\title{
The NASA Astrophysics Data System: Data holdings
}

\author{
Carolyn S. Grant, Alberto Accomazzi, Guenther Eichhorn, Michael J. Kurtz, and Stephen S. Murray \\ Harvard-Smithsonian Center for Astrophysics, Cambridge, MA 02138, U.S.A.
}

Received August 30; accepted September 3, 1999

\begin{abstract}
Since its inception in 1993, the ADS Abstract Service has become an indispensable research tool for astronomers and astrophysicists worldwide. In those seven years, much effort has been directed toward improving both the quantity and the quality of references in the database. From the original database of approximately 160000 astronomy abstracts, our dataset has grown almost tenfold to approximately 1.5 million references covering astronomy, astrophysics, planetary sciences, physics, optics, and engineering. We collect and standardize data from approximately 200 journals and present the resulting information in a uniform, coherent manner. With the cooperation of journal publishers worldwide, we have been able to place scans of full journal articles on-line back to the first volumes of many astronomical journals, and we are able to link to current version of articles, abstracts, and datasets for essentially all of the current astronomy literature. The trend toward electronic publishing in the field, the use of electronic submission of abstracts for journal articles and conference proceedings, and the increasingly prominent use of the World Wide Web to disseminate information have enabled the ADS to build a database unparalleled in other disciplines.

The ADS can be accessed at:

http://adswww.harvard.edu
\end{abstract}

Key words: methods: data analysis - astronomical bibliography

\section{Introduction}

Astronomers today are more prolific than ever before. Studies in publication trends in astronomy (Abt 1994; Abt 1995; Schulman et al. 1997) have hypothesized that the current explosion in published papers in astronomy is due to a combination of factors: growth in professional

Send offprint requests to: C.S. Grant,

e-mail: cgrant@cfa.harvard.edu society membership, an increase in papers by multiple authors, the launching of new spacecrafts, and increased competition for jobs and PIs in the field (since candidate evaluation is partially based on publication history). As the number of papers in the field grows, so does the need for tools which astronomers can use to locate that fraction of papers which pertain to their specific interests.

The ADS Abstract Service is one of several bibliographic services which provide this function for astronomy, but due to the broad scope of our coverage and the simplicity of access to our data, astronomers now rely extensively on the ADS. Other bibliographic services not only link to us, but some have built their bibliographic search capabilities on top of the ADS system. The International Society for Optical Engineering (SPIE) and the NASA Technical Report Service (NTRS) are two such services.

The evolution of the Astrophysics Data System (ADS) has been largely data-driven. Our search tools and indexing routines have been modified to maximize speed and efficiency based on the content of our dataset. As new types of data (such as electronic versions of articles) became available, the Abstract Service quickly incorporated that new feature. The organization and standardization of the database content is the very core upon which the Abstract Service has been built.

This paper contains a description of the ADS Abstract Service from a "data" point of view, specifically descriptions of our holdings and of the processes by which we ingest new data into the system. Details are provided on the organization of the databases (Sect. 2), the description of the data in the databases (Sect. 3), the creation of bibliographic records (Sect. 4), the procedures for updating the database (Sect. 5), and on the scanned articles in the Astronomy database (Sect. 6). We discuss the interaction between the ADS and the journal publishers (Sect. 7) and analyze some of the numbers corresponding to the datasets (Sect. 8). In conjunction with three other ADS papers in this volume, this paper is intended to offer details on the entire Abstract Service with the hopes 
that astronomers will have a better understanding of the reference data upon which they rely for their research. In addition, we hope that researchers in other disciplines may be able to benefit from some of the details described herein.

As is often the case with descriptions of active Internet resources, what follows is a description of the present situation with the ADS Abstract Service. New features are always being added, some of which necessitate changes in our current procedures. Furthermore, with the growth of electronic publishing, some of our core ideas about bibliographic tools and requirements must be reconsidered in order to be able to take full advantage of new publishing technologies for a new millennium.

\section{The databases}

The ADS Abstract Service was originally conceived of in the mid 1980's as a way to provide on-line access to bibliographies of astronomers which were previously available only through expensive librarian search services or through the A\&A Abstracts series (Schmadel 1979; Schmadel 1982; Schmadel 1989), published by the Astronomisches Rechen-Institut in Heidelberg. While the ideas behind the Abstract Service search engine were being developed (see Kurtz et al. 2000, hereafter OVERVIEW), concurrent efforts were underway to acquire a reliable data source on which to build the server. In order to best develop the logistics of the search engine it was necessary to have access to real literature data from the past and present, and to set up a mechanism for acquiring data in the future.

An electronic publishing meeting in the spring of 1991 brought together a number of organizations whose ultimate cooperation would be necessary to make the system a reality (see OVERVIEW for details). NASA's Scientific and Technical Information Program (STI) offered to provide abstracts to the ADS. STI's abstracts were a rewritten version of the original abstracts, categorized and keyworded by professional editors. They not only abstracted the astronomical literature, but many other scientific disciplines as well. With STI agreeable to providing the past and present literature, and the journals committed to providing the future literature, the data behind the system fell into place. The termination of the journal abstracting by the STI project several years later was unfortunate, but did not cause the collapse of the ADS Abstract Service because of the commitment of the journal publishers to distribute their information freely.

The STI abstracting approximately covered the period from 1975 to 1995 . With the STI data alone, we estimated the completeness of the Astronomy database to be better than $90 \%$ for the core astronomical journals. Fortunately, with the additional data supplied by the journals, by SIMBAD (Set of Identifications,
Measurements, and Bibliographies for Astronomical Data, Egret \& Wenger 1988) at the CDS (Centre de Données Astronomiques de Strasbourg), and by performing Optical Character Recognition (OCR) on the scanned table of contents (see Sect. 6 below), we are now closer to $99 \%$ complete for that period. In the period since then we are $100 \%$ complete for those journals which provide us with data, and significantly less complete for those which do not (e.g. many observatory publications and non-U.S. journals). The data prior to 1975 are also significantly incomplete, although we are currently working to improve the completeness of the early data, primarily through scanning the table of contents for journal volumes as they are placed on-line. We are $100 \%$ complete for any journal volume which we have scanned and put on-line, since we verify that we have all bibliographic entries during the procedure of putting scans on-line.

Since the STI data were divided into categories, it was easy to create additional databases with non-astronomical data which were still of interest to astronomers. The creation of an Instrumentation database has enabled us to provide a database for literature related to astronomical instrumentation, of particular interest to those scientists building astronomical telescopes and satellite instruments. We were fortunate to get the cooperation of the SPIE very quickly after releasing the Instrumentation database. SPIE has become our major source of abstracts for the Instrumentation database now that STI no longer supplies us with data.

Our Physics and Geophysics database, the third database to go on-line, is intended for scientists working in physics-related fields. We add authors and titles from all of the physics journals of the American Institute of Physics (AIP), the Institute of Physics (IOP), and the American Physical Society (APS), as well as many physics journals from publishers such as Elsevier and Academic Press (AP).

The fourth database in the system, the Preprint database, contains a subset of the Los Alamos National Laboratory's (LANL) Preprint Archive (Los Alamos National Laboratory 1991). Our database includes the LANL astro-ph preprints which are retrieved from LANL and indexed nightly through an automated procedure. That dataset includes preprints from astronomical journals submitted directly by authors.

\section{Description of the data}

The original set of data from STI contained several basic fields of data (author, title, keywords, and abstracts) to be indexed and made available for searching. All records were keyed on STI's accession number, a nine-digit code consisting of a letter prefix (A or N) followed by a twodigit publication year, followed by a five-letter identifier (e.g. A95-12345). Data were stored in files named by accession number. 
With the inclusion of data from other sources, primarily the journal publishers and SIMBAD, we extended STI's concept of the accession number to handle other abstracts as well. Since the ADS may receive the same abstract from multiple sources, we originally adopted a system of using a different prefix letter with the remainder of the accession number being the same to describe abstracts received from different sources. Thus, the same abstract for the above accession number from STI would be listed as J95-12345 from the journal publisher and S9512345 from SIMBAD. This allowed the indexing routines to consider only one instance of the record when indexing. Recently, limitations in the format of accession numbers and the desire to index data from multiple sources (rather than just STI's version) have prompted us to move to a data storage system based entirely on the bibliographic code.

\subsection{Bibliographic codes}

The concept of a unique bibliographic code used to identify an article was originally conceived of by SIMBAD and NED (NASA's Extragalactic Database, Helou \& Madore 1988). The original specification is detailed in Schmitz et al. (1995). In the years since, the ADS has adopted and expanded their definition to be able to describe references outside of the scope of those projects.

The bibliographic code is a 19-character string comprised of several fields which usually enables a user to identify the full reference from that string. It is defined as follows:

\section{YYYYJJJJJVVVVMPPPPA}

where the fields are defined in Table 1.

The journal field is left-justified and the volume and page fields are right-justified. Blank spaces and leading zeroes are replaced by periods. For articles with page numbers greater than 9999, the M field contains the first digit of the page number.

Creating bibliographic codes for the astronomical journals is uncontroversial. Each journal typically has a commonly-used abbreviation, and the volume and page are easily assigned (e.g. 1999PASP..111..438F). Each volume tends to have individual page numbering, and in those cases where more than one article appears on a page (such as errata), a "Q", "R", "S", etc. is used as the qualifier for publication to make bibliographic codes unique. When page numbering is not continuous across issue numbers (such as Sky \& Telescope), the issue number is represented by a lower case letter as the qualifier for publication (e.g. "a" for issue 1). This is because there may be multiple articles in a volume starting on the same page number.

Creating bibliographic codes for the "grey" literature such as conference proceedings and technical reports is a more difficult task. The expansion into these additional types of data included in the ADS required us to modify the original prototype bibliographic code definition in order to present identifiers which are easily recognizable to the user. The prototype definition of the bibliographic code suggested using a single letter in the second place of the volume field to identify non-standard references (catalogs, $\mathrm{PhD}$ theses, reports, preprints, etc.) and using the third and fourth place of that field to unduplicate and report volume numbers (e.g. 1981CRJS..R.3...14W). Since we felt that this created codes unidentifiable to the typical user and since NED and SIMBAD did not feel that users needed to be able to identify books directly from their bibliographic codes, the ADS adopted different rules for creating codes to identify the grey literature.

It is straightforward to create bibliographic codes for conference proceedings which are part of a series. For example, the IAU Symposia Series (IAUS) contains volume numbers and therefore fits the journal model for bibliographic codes. Other conference proceedings, books, colloquia, and reports in the ADS typically contain a four letter word in the volume field such as "conf", "proc", "book", "coll", or "rept". When this is the case with a bibliographic code, the journal field typically consists of the first letter from important words in the title. This can give the user the ability to identify a conference proceeding at a glance (e.g. "ioda.book" for "Information and On-Line Data in Astronomy"). We will often leave the fifth place of the journal field as a dot for "readability" (e.g. 1995ioda.book..175M). For most proceedings which are also published as part of a series (e.g. ASP Conference Series, IAU Colloquia, AIP Conference Series), we include in the system two bibliographic codes, one as described above and one which contains the series name and the volume (see Sect. 5.1). We do this so that users can see, for example, that a paper published in one of the "Astronomical Data Analysis Software and Systems" series is clearly labelled as "adass" whereas a typical user might not remember which volume of ASPC contained those ADASS papers. This increases the user's readability of bibliographic codes.

With the STI data, the details were often unclear as to whether an article was from a conference proceeding, a meeting, a colloquium, etc. We assigned those codes as best we could, making no significant distinction between them. For conference abstracts submitted by the editors of a proceedings prior to publication, we often do not have page numbers. In this case, we use a counter in lieu of a page number and use an "E" (for "Electronic") in the fourteenth column, the qualifier for publication. If these conference abstracts are then published, their bibliographic codes are replaced by a bibliographic code complete with page number. If the conference abstracts are published only on-line, they retain their electronic bibliographic code with its $\mathrm{E}$ and counter number. 
Table 1. Bibliographic code definition (e.g. 1996A\&AS..115....1S)

\begin{tabular}{lll}
\hline Field & Definition & Example \\
\hline YYYY & Publication Year & 1997 \\
JJJJJ & Journal Abbreviation & ApJ, A\&A, MNRAS, etc. \\
VVVV & Volume Number & 480 \\
M & Qualifier for Publication & L (for Letter), P (for Pink Page) \\
& & Q, R, S, etc. for unduplicating \\
& & $\mathrm{a}, \mathrm{b}, \mathrm{c}$, etc. for issue number \\
PPPP & Page Number & 129 \\
A & First Letter of the First Author's Surname & $\mathrm{N}$ \\
\hline
\end{tabular}

There are several other instances of datasets where the bibliographic codes are non-standard. PhD theses in the system use "PhDT" as the journal abbreviation, contain no volume number, and contain a counter in lieu of a page number. Since $\mathrm{PhD}$ theses, like all bibliographic codes, are unique across all of the databases, the counter makes the bibliographic code an identifier for only one thesis. IAU Circulars also use a counter instead of a page number. Current Circulars are electronic in form, and although not technically a new page, the second item of an IAU Circular is the electronic equivalent of a second page. Using the page number as a counter enables us to minimize use of the $\mathrm{M}$ identifier in the fourteenth place of a bibliographic code for unduplicating. This is desirable since codes containing those identifiers are essentially impossible to create a priori, either by the journals or by users.

The last set of data currently included in the ADS which contain non-standard bibliographic codes is the "QB" book entries from the Library of Congress. QB is the Library of Congress code for astronomy-related books and we have put approximately 17000 of these references in the system. Because the QB numbers are identifiers by themselves, we have made an exception to the bibliographic code format to use the QB number (complete with any series or part numbers), prepended with the publication year as the bibliographic code. Such an entry is easily identifiable as a book, and these codes enable users to locate the books in most libraries.

It is worth noting that while the bibliographic code makes identification simple for the vast majority of references in the system, we are aware of two instances where the bibliographic definition breaks down. The use of the fourteenth column for a qualifier such as "L" for $A p J$ Letters makes it impossible to use that column for unduplicating. Therefore, if there are two errata on the same page with the same author initial, there is no way to create unique bibliographic codes for them. We are aware of only one such instance in the 33 years of publication of ApJ Letters. Second, with the electronic publishing of an increasing number of journals, the requirement of page numbers to locate articles becomes unnecessary. The journal Physical Review $D$ is currently using 6-digit article identifiers as page numbers. Since the bibliographic code allows for page numbers not longer than 5 digits, we are currently converting these 6-digit identifiers to their 5digit hexagesimal equivalent. Both of these anomalies indicate that over the next few years we will likely need to alter the current bibliographic definition in order to allow consistent identification of articles for all journals.

\subsection{Data fields}

The databases are set up such that some data fields are searchable and others are not. The searchable fields (title, author, and text) are the bulk of the important data, and these fields are indexed so that a query to the database returns the maximum number of meaningful results. (see Accomazzi et al. 2000, hereafter ARCHITECTURE). The text field is the union of the abstract, title, keywords, and comments. Thus, if a user requests a particular word in the text field, all papers are returned which contain that word in the abstract OR in the title $\mathbf{O R}$ in the keywords OR in the comments. Appendix A shows version 1.0 of the Extensible Markup Language (XML, see Sect. 3.4) Document Type Definition (DTD) for text files in the ADS Abstract Service. The DTD lists fields currently used or expected to be used in text files in the ADS (see Sect. 5.2 for details on the text files). We intend to reprocess the current journal and affiliation fields in order to extract some of these fields.

Since STI ceased abstracting the journal literature, we decided to make the keywords themselves no longer a searchable entity for the time being - they are searchable only through the abstract text field. STI used a different standard set of keywords from the AAS journals, who use a different set of keywords from AIP journals (e.g. AJ prior to 1998). In addition, keywords from a single journal such as the Astrophysical Journal (ApJ) have evolved over the years so that early $A p J$ volume keywords are not consistent with later volumes. In order to build one coherent set of keywords, an equivalence or synonym table for these different keyword sets must be created. We are investigating different schemes for doing this, and currently plan to have a searchable keyword field again, which encompasses all keywords in the system and equates those from different keyword systems which are similar (Lee et al. 1999). 
The current non-searchable fields in the ADS databases include the journal field, author affiliation, category, abstract copyright, and abstract origin. Although we may decide to create an index and search interface for some of these entities (such as category), others will continue to remain unsearchable since searching them is not useful to the typical user. In particular, author affiliations would be useful to search, however this information is inconsistently formatted so it is virtually impossible to collect all variations of a given institution for indexing coherently. Furthermore, we have the author affiliations for only about half of the entries in the Astronomy database so we have decided to keep this field non-searchable. For researchers wishing to analyze affiliations on a large scale, we can provide this information on a collaborative basis.

\subsection{Data sources}

The ADS currently receives abstracts or table of contents (ToC) references from almost two hundred journal sources. Tables 2, 3, and 4 list these journals, along with their bibliographic code abbreviation, source, frequency with which we receive the data, what data are received, and any links we can create to the data. ToC references typically contain only author and title, although sometimes keywords are included as well. The data are contributed via email, $\mathrm{ftp}$, or retrieved from web sites around the world at a frequency ranging from once a week to approximately once a year. The term "often" used in the frequency column implies that we get them more frequently than once a month, but not necessarily on a regular basis. The term "occasionally" is used for those journals who submit data to us infrequently.

Updates to the Astronomy and Instrumentation databases occur approximately every two weeks, or more often if logistically possible, in order to keep the database current. Recent enhancements to the indexing software have enabled us to perform instantaneous updates, triggered by an email containing new data (see ARCHITECTURE). Updates to the Physics database occurs approximately once every two months. As stated earlier, the Preprint database is updated nightly.

\subsection{Data formats}

The ADS is able to benefit from certain standards which are adhered to in the writing and submission practices of astronomical literature. The journals share common abbreviations and text formatting routines which are used by the astronomers as well. The use of TeX (Knuth 1984) and LaTeX (Lamport 1986), and their extension to BibTeX (Lamport 1986) and AASTeX (American Astronomical Society 1999) results in common formats among some of our data sources. This enables the reuse of parsing routines to convert these formats to our standard format. Other variations of TeX used by journal publishers also allows us to use common parsing routines which greatly facilitates data loading.

TeX is a public domain typesetting program designed especially for math and science. It is a markup system, which means that formatting commands are interspersed with the text in the TeX input file. In addition to commands for formatting ordinary text, TeX includes many special symbols and commands with which you can format mathematical formulae with both ease and precision. Because of its extraordinary capabilities, TeX has become the leading typesetting system for science, mathematics, and engineering. It was developed by Donald Knuth at Stanford University.

LaTeX is a simplified document preparation system built on TeX. Because LaTeX is available for just about any type of computer and because LaTeX files are ASCII, scientists are able to send their papers electronically to colleagues around the world in the form of LaTeX input. This is also true for other variants of TeX, although the astronomical publishing community has largely centered their publishing standards on LaTeX or one of the software packages based on LaTeX, such as BibTeX or AASTeX. BibTeX is a program and file format designed by Oren Patashnik and Leslie Lamport in 1985 for the LaTeX document preparation system, and AASTeX is a LaTeXbased package that can be used to mark up manuscripts specifically for American Astronomical Society (AAS) journals.

Similar to the widespread acceptance of TeX and its variants, the extensive use of SGML (Standard Generalized Markup Language, Goldfarb \& Rubinsky 1991) by the members of the publishing community has given us the ability to standardize many of our parsing routines. All data gleaned off the World Wide Web share features due to the use of HTML (HyperText Markup Language, Powell \& Whitworth 1998), an example of SGML. Furthermore, the trend towards using XML (Extensible Markup Language, Harold 1999) to describe text documents will enable us to share standard document attributes with other members of the astronomical community. XML is a subset of SGML which is intended to enable generic SGML to be served, received, and processed on the Web in the way that is now possible with HTML. The ADS parsing routines benefit from these standards in several ways: we can reuse routines designed around these systems; we are able to preserve original text representations of entities such as embedded accents so these entities are displayed correctly in the user's browser; and we are able to capture value-added features such as electronic URLs and email addresses for use elsewhere in our system.

In order to facilitate data exchange between different parts of the ADS, we make use of a tagged format similar 
Table 2. The ADS astronomy database

\begin{tabular}{|c|c|c|c|c|c|}
\hline Journal & Source & Full Name & How Often & Kind of Data & Links $^{\mathrm{a}}$ \\
\hline $\mathrm{A} \& \mathrm{~A}$ & Springer & Astronomy \& Astrophysics & $3 \times$ month & abstracts & $\mathrm{E}, \mathrm{F}$ \\
\hline $\mathrm{A} \& \mathrm{ARv}$ & Springer & Astronomy \& Astrophysics Review & occasionally & abstracts & $\mathrm{F}$ \\
\hline $\mathrm{A} \& \mathrm{AS}$ & EDP Sciences & Astronomy \& Astrophysics Supplement & $2 \times$ month & abstracts & $\mathrm{E}, \mathrm{F}, \mathrm{R}$ \\
\hline AcA & AcA & Acta Astronomica & $4 \times$ year & abstracts & $\mathrm{G}$ \\
\hline ADIL & NCSA ADIL & Astronomy Data Image Library & occasionally & abstracts & $\mathrm{D}$ \\
\hline AdSpR & Elsevier & Advances in Space Research & often & abstracts & \\
\hline AGAb & $\mathrm{AG}^{\mathrm{b}}$ & Astronomische Gesellschaft Abstracts & occasionally & abstracts & \\
\hline AJ & $\mathrm{UCP}^{\mathrm{c}}$ & Astronomical Journal & monthly & abstracts & $\mathrm{E}, \mathrm{F}, \mathrm{R}$ \\
\hline $\mathrm{AN}$ & $\mathrm{AN}$ & Astronomische Nachrichten & bimonthly & abstracts & \\
\hline Ap\&SS & Kluwer & Astrophysics and Space Science & often & abstracts & \\
\hline $\mathrm{APh}$ & Elsevier & Astroparticle Physics & bimonthly & abstracts & $\mathrm{E}$ \\
\hline ApJ & $\mathrm{UCP}$ & Astrophysical Journal & $3 \times$ month & abstracts & $\mathrm{E}, \mathrm{F}, \mathrm{R}$ \\
\hline ApJL & $\mathrm{UCP}$ & Astrophysical Journal Letters & $3 \times$ month & abstracts & $\mathrm{E}, \mathrm{F}, \mathrm{R}$ \\
\hline ApJS & $\mathrm{UCP}$ & Astrophysical Journal Supplement & monthly & abstracts & $\mathrm{E}, \mathrm{F}, \mathrm{R}$ \\
\hline ARA\&A & AnnRev & Annual Review of Astronomy and Astrophysics & $1 \times$ year & abstracts & $\mathrm{E}, \mathrm{F}$ \\
\hline AREPS & AnnRev & Annual Review of Earth and Planetary Sciences & $1 \times$ year & abstracts & $\mathrm{E}, \mathrm{F}$ \\
\hline ARep & $\mathrm{AIP}^{\mathrm{d}}$ & Astronomy Reports & bimonthly & abstracts & M \\
\hline AstL & AstL & Astronomy Letters & bimonthly & abstracts & M \\
\hline ATel & ATel & The Astronomer's Telegram & often & abstracts & $\mathrm{E}$ \\
\hline AVest & AVest & Astronomicheskii Vestnik & bimonthly & abstracts & \\
\hline BAAS & AAS & Bulletin of the American Astronomical Society & $2 \times$ year $($ AAS $)$ & abstracts & \\
\hline BAAS & AAS & Bulletin of the American Astronomical Society & $1 \times$ year $(\mathrm{DDA})$ & abstracts & \\
\hline BAAS & AAS & Bulletin of the American Astronomical Society & $1 \times$ year $(\mathrm{DPS})$ & abstracts & \\
\hline BAAS & AAS & Bulletin of the American Astronomical Society & $1 \times$ year $(\mathrm{HEA})$ & abstracts & \\
\hline BAAS & AAS & Bulletin of the American Astronomical Society & $1 \times$ year $(\mathrm{SPD})$ & abstracts & \\
\hline BaltA & BaltA & Baltic Astronomy & $4 \times$ year & abstracts & $\mathrm{R}$ \\
\hline $\mathrm{BeSN}$ & $\mathrm{BeSN}$ & BE Star Newsletter & occasionally & $\mathrm{ToC}$ & G \\
\hline BOBeo & BOBeo & Bulletin Astronomique de Belgrade & occasionally & abstracts & $\mathrm{G}$ \\
\hline Books & CUP & Cambridge University Press & occasionally & $\mathrm{ToC}$ & \\
\hline Books & $\mathrm{LOC}$ & Library of Congress & occasionally & abstracts & $\mathrm{L}$ \\
\hline Books & Springer & Springer Verlag & occasionally & $\mathrm{ToC}$ & \\
\hline Books & USci & University Science Books Publishers & occasionally & abstracts & M \\
\hline Books & Wiley & Wiley Publishers & occasionally & abstracts & \\
\hline CeMDA & Kluwer & Celestial Mechanics and Dynamical Astronomy & often & abstracts & \\
\hline ChA\&A & Elsevier & Chinese Astronomy and Astrophysics & $4 \times$ year & $\mathrm{ToC}$ & \\
\hline CoKon & Konkoly & Communications of the Konkoly Observatory & occasionally & abstracts & $\mathrm{G}$ \\
\hline Conferences & Boon & Priscilla Boon, Conference Proceedings & occasionally & $\mathrm{ToC}$ & \\
\hline Conferences & Editors & Conference Proceeding Editor Submissions & often & abstracts & \\
\hline Conferences & ESO & European Southern Observatory Library & monthly & $\mathrm{ToC}$ & \\
\hline Conferences & LPI & Lunar and Planetary Institute Proceedings & occasionally & abstracts & $\mathrm{F}$ \\
\hline Conferences & STSci & Space Telescope Science Institute Library & monthly & $\mathrm{ToC}$ & \\
\hline Conferences & UTAL & University of Toronto Library & weekly & ToC & \\
\hline CoSka & CoSka & Contributions from the Ast. Obs. Skalnate Pleso & occasionally & abstracts & $\mathrm{G}$ \\
\hline DSSN & DSSN & Delta Scuti Star Newsletter & occasionally & abstracts & $\mathrm{E}$ \\
\hline DyAtO & Elsevier & Dynamics of Atmospheres and Oceans & occasionally & $\mathrm{ToC}$ & \\
\hline E\&PSL & Elsevier & Earth \& Planetary Science Letters & occasionally & $\mathrm{ToC}$ & $\mathrm{E}, \mathrm{D}$ \\
\hline EM\&P & Kluwer & Earth, Moon, and Planets & often & abstracts & \\
\hline ESRv & Elsevier & Earth Science Reviews & occasionally & $\mathrm{ToC}$ & \\
\hline ExA & Kluwer & Experimental Astronomy & occasionally & abstracts & \\
\hline $\mathrm{FCPh}$ & $\mathrm{OPA}^{\mathrm{e}}$ & Fundamentals of Cosmic Physics & occasionally & abstracts & \\
\hline $\mathrm{GeCoA}$ & Elsevier & Geochimica et Cosmochimica Acta & often & $\mathrm{ToC}$ & \\
\hline GeoRL & $\mathrm{AGU}^{\mathrm{f}}$ & Geophysical Research Letters & $2 \times$ month & $\mathrm{ToC}$ & $\mathrm{E}, \mathrm{F}$ \\
\hline GeoJI & Blackwell & Geophysical Journal International & $2 \times$ month & abstracts & $\mathrm{E}, \mathrm{F}, \mathrm{R}$ \\
\hline GReGr & Plenum & General Relativity and Gravitation & monthly & abstracts & \\
\hline IAUC & $\mathrm{CBAT}^{\mathrm{g}}$ & IAU Circulars & weekly & abstracts & $\mathrm{E}$ \\
\hline IBVS & Konkoly & Information Bulletin on Variable Stars & often & abstracts & $\mathrm{E}, \mathrm{F}$ \\
\hline Icar & $\mathrm{AP}^{\mathrm{h}}$ & Icarus & monthly & abstracts & $\mathrm{E}, \mathrm{F}, \mathrm{R}$ \\
\hline
\end{tabular}


Table 2. continued

\begin{tabular}{|c|c|c|c|c|c|}
\hline Journal & Source & Full Name & How Often & Kind of Data & $\operatorname{Links}^{\mathrm{a}}$ \\
\hline IrAJ & Ir AJ & Irish Astronomical Journal & $2 \times$ year & abstracts & \\
\hline JASS & JASS & Journal of Astronomy and Space Sciences & occasionally & abstracts & $\mathrm{F}$ \\
\hline JAVSO & AAVSO & Journal of the A.A.V.S.O. & occasionally & abstracts & \\
\hline JBAA & BAA & Journal of the British Astronomical Association & bimonthly & abstracts & \\
\hline JIMO & IMO & Journal of the International Meteor Organization & occasionally & abstracts & \\
\hline JGR & AGU & Journal of Geophysical Research A (Space Physics) & monthly & $\mathrm{ToC}$ & \\
\hline JGR & AGU & Journal of Geophysical Research E (Planets) & monthly & $\mathrm{ToC}$ & \\
\hline JKAS & KAS & Journal of the Korean Astronomical Society & occasionally & abstracts & \\
\hline JRASC & RASC & Journal of the Royal Astronomical Society of Canada & occasionally & abstracts & \\
\hline M\&PS & $\mathrm{M} \& \mathrm{PS}$ & Meteoritics \& Planetary Science & bimonthly & abstracts & \\
\hline MNRAS & Blackwell & Monthly Notices of the Royal Astronomical Society & $3 \times$ month & abstracts & $\mathrm{E}, \mathrm{F}, \mathrm{R}$ \\
\hline MPEC & CBAT & Minor Planet Electronic Circulars & weekly & abstracts & $\mathrm{E}$ \\
\hline Nature & Nature & Nature & weekly & abstracts & \\
\hline NewA & Elsevier & New Astronomy & often & abstracts & $\mathrm{E}$ \\
\hline NewAR & Elsevier & New Astronomy Reviews (formerly VA) & occasionally & abstracts & \\
\hline OAP & OAP & Odessa Astronomical Publications & occasionally & abstracts & \\
\hline Obs & Obs & The Observatory & occasionally & $\mathrm{ToC}$ & \\
\hline P\&SS & Elsevier & Planetary and Space Science & monthly & $\mathrm{ToC}$ & \\
\hline PASA & PASA & Publications of the Astronomical Society of Australia & $2 \times$ year & abstracts & $\mathrm{E}, \mathrm{F}$ \\
\hline PASJ & PASJ & Publications of the Astronomical Society of Japan & bimonthly & abstracts & $\mathrm{R}$ \\
\hline PASP & $\mathrm{UCP}$ & Publications of the Astronomical Society of the Pacific & monthly & abstracts & $\mathrm{E}, \mathrm{F}, \mathrm{R}$ \\
\hline PDS & PDS & Planetary Data System & occasionally & abstracts & $\mathrm{P}$ \\
\hline PEPI & Elsevier & Physics of the Earth and Planetary Interiors & monthly & $\mathrm{ToC}$ & \\
\hline PhDT & UMass & University of Massachusetts & occasionally & abstracts & $\mathrm{D}$ \\
\hline PhDT & UMI & University Microfilm, Inc. & occasionally & abstracts & M \\
\hline PKAS & KAS & Publications of the Korean Astronomical Society & occasionally & abstracts & \\
\hline RvMA & $\mathrm{AG}$ & Reviews of Modern Astronomy & occasionally & $\mathrm{ToC}$ & \\
\hline $\mathrm{RMxAC}$ & $\mathrm{UNAM}^{\mathrm{i}}$ & Revista Mexicana Conference Series & occasionally & $\mathrm{ToC}$ & \\
\hline $\mathrm{S} \& \mathrm{~T}$ & Sky Publishing & Sky \& Telescope & $2 \times$ year & $\mathrm{ToC}$ & \\
\hline Sci & Science & Science & weekly & $\mathrm{ToC}$ & $\mathrm{E}$ \\
\hline $\mathrm{SoPh}$ & Stet. & Solar Physics & often & abstracts & \\
\hline $\mathrm{SSRv}$ & Kluwer & Space Science Reviews & often & abstracts & \\
\hline VA & Elsevier & Vistas in Astronomy & occasionally & $\mathrm{ToC}$ & \\
\hline Various & $\mathrm{ARI}^{\mathrm{j}}$ & Veröffentlichungen ARI & occasionally & $\mathrm{ToC}$ & $\mathrm{L}$ \\
\hline Various & Authors & Author Submissions & often & abstracts & \\
\hline Various & Knudsen & Helen Knudsen's Monthly Index of Astronomy & occasionally & $\mathrm{ToC}$ & \\
\hline Various & NED & NASA Extragalactic Database & occasionally & $\mathrm{ToC}$ & $\mathrm{N}$ \\
\hline Various & SIMBAD & SIMBAD & $2 \times$ month & $\mathrm{ToC}$ & $\mathrm{D}, \mathrm{S}$ \\
\hline Various & STI & NASA's Science and Technical Index & $2 \times$ month & abstracts & \\
\hline Various & USNO & United States Naval Observatory Library & occasionally & $\mathrm{ToC}$ & \\
\hline
\end{tabular}

${ }^{\text {a }}$ Letter codes describing what data are available.

b Astronomische Gesellschaft.

${ }^{\mathrm{c}}$ University of Chicago Press.

d American Institute of Physics.

e Overseas Publishers Association.

${ }^{\mathrm{f}}$ American Geophysical Union.

g Central Bureau for Astronomical Telegrams.

h Academic Press.

${ }^{\text {i }}$ Universitad Nacional Autonoma de Mexico.

j Astronomisches Rechen-Institut. 
Table 3. The ADS instrumentation database

\begin{tabular}{|c|c|c|c|c|c|}
\hline Journal & Source & Full Name & How Often & Kind of Data & $\operatorname{Links}^{\mathrm{a}}$ \\
\hline ACAau & Elsevier & Acta Astronautica & often & ToC & \\
\hline ApOpt & $\mathrm{OSA}^{\mathrm{b}}$ & Applied Optics & often & abstracts & M \\
\hline ApScR & Kluwer & Applied Scientific Research & occasionally & $\mathrm{ToC}$ & \\
\hline ChJLB & OSA & Chinese Journal of Lasers B & occasionally & abstracts & \\
\hline IJQE & OSA & Journal of Quantum Electronics & often & abstracts & \\
\hline JBO & $\mathrm{SPIE}^{\mathrm{c}}$ & Journal of Biomedical Optics & occasionally & abstracts & \\
\hline JEI & SPIE & Journal of Electronic Imaging & occasionally & abstracts & \\
\hline JEnMa & Kluwer & Journal of Engineering Mathematics & occasionally & ToC & \\
\hline JMiMi & $\mathrm{IOP}^{\mathrm{d}}$ & Journal of Micromechanics \& Microengineering & often & $\mathrm{ToC}$ & $\mathrm{E}$ \\
\hline $\mathrm{JO}$ & IOP & Journal of Optics & often & $\mathrm{ToC}$ & $\mathrm{E}$ \\
\hline JOptT & OSA & Journal of Optical Technology & often & abstracts & M \\
\hline JVST & AIP & Journal of Vacuum \& Science Technology & often & $\mathrm{ToC}$ & $\mathrm{M}$ \\
\hline OptCo & Elsevier & Optics Communications & often & abstracts & \\
\hline OptEn & SPIE & Optical Engineering & often & abstracts & \\
\hline OptFT & AP & Optical Fiber Technology & often & abstracts & \\
\hline OptL & OSA & Optics Letters & often & $\mathrm{ToC}$ & $\mathrm{M}$ \\
\hline OptLE & Elsevier & Optics and Lasers in Engineering & bimonthly & $\mathrm{ToC}$ & \\
\hline OptLT & Elsevier & Optics \& Laser Technology & occasionally & ToC & \\
\hline OptPN & AIP & Optics \& Photonics News & often & abstracts & M \\
\hline OptSp & OSA & Optics and Spectroscopy & often & abstracts & M \\
\hline OSAJ & AIP & Journal of the Optical Society of America A & often & abstracts & $\mathrm{M}$ \\
\hline OSAJB & AIP & Journal of the Optical Society of America B & often & abstracts & $\mathrm{M}$ \\
\hline $\mathrm{PApO}$ & IOP & Pure Applied Optics & often & $\mathrm{ToC}$ & $\mathrm{E}$ \\
\hline $\operatorname{PrAeS}$ & Elsevier & Progress in Aerospace Sciences & occasionally & $\mathrm{ToC}$ & \\
\hline $\mathrm{RScI}$ & AIP & Review of Scientific Instruments & often & ToC & $\mathrm{M}$ \\
\hline SPIE & SPIE & SPIE Proceedings & often & abstracts & $\mathrm{M}$ \\
\hline
\end{tabular}

${ }^{a}$ Letter codes describing what data are available.

${ }^{\mathrm{b}}$ Optical Society of America.

c The International Society for Optical Engineering (SPIE).

${ }^{\mathrm{d}}$ Institute of Physics.

to the "Refer" format (Jacobsen 1996). Refer is a preprocessor for the word processors nroff and troff which finds and formats references. While our tagged formats share some common fields $(\% \mathrm{~A}, \% \mathrm{~T}, \% \mathrm{~J}, \% \mathrm{D})$, the Refer format is not specific enough to be used for our purposes. Items such as objects, URLs and copyright notices are beyond the scope of the Refer syntax. Details on our tagged format are provided in Table 5. Reading and writing routines for this format are shared by loading and indexing routines, and a number of our data sources submit abstracts to us in this format.

\section{Creating the bibliographic records}

One of the basic principles in the parsing and formatting of the bibliographic data incorporated into the ADS database over the years has been to preserve as much of the original information as possible and delay any syntactic or semantic interpretation of the data until a later stage. From the implementation point of view, this means that bibliographic records provided to the ADS by publishers or other data sources typically are saved as files which are tagged with their origin, entry date, and any other ancillary information relevant to their contents (e.g. if the fields in the record contain data which was transliterated or converted to ASCII).

For instance, the records provided to the ADS by the University of Chicago Press (the publisher of several major U.S. astronomical journals) are SGML documents which contain a unique manuscript identifier assigned to the paper during the electronic publishing process. This identifier is saved in the file created by the ADS system for this bibliographic entry.

Because data about a particular bibliographic entry may be provided to the ADS by different sources and at different times, we adopted a multi-step procedure in the creation and management of bibliographic records:

1) Tokenization: Parsing input data into a memoryresident data structure using procedures which are formatand source-specific;

2) Identification: Computing the unique bibliographic record identifier used by the ADS to refer to this record;

3) Instantiation: Creating a new record for each bibliography formatted according to the ADS "standard" format;

4) Extraction: Selecting the best information from the different records available for the same bibliography and 
Table 4. The ADS physics database

\begin{tabular}{|c|c|c|c|c|c|}
\hline Journal & Source & Full Name & How Often & Kind of Data & $\operatorname{Links}^{\mathrm{a}}$ \\
\hline AcPhy & AIP & Acoustical Physics & occasionally & ToC & $\mathrm{M}$ \\
\hline ADNDT & $\mathrm{AP}$ & Atomic Data and Nuclear Data Tables & occasionally & abstracts & \\
\hline AnPhy & $\mathrm{AP}$ & Annals of Physics & often & abstracts & \\
\hline ApPhL & AIP & Applied Physics Letters & often & ToC & $\mathrm{M}$ \\
\hline ASAJ & AIP & Journal of the Acoustical Society of America & often & ToC & $\mathrm{M}$ \\
\hline Chaos & AIP & Chaos & occasionally & ToC & $\mathrm{M}$ \\
\hline ComPh & AIP & Computers In Physics & occasionally & $\mathrm{ToC}$ & M \\
\hline CQGra & IOP & Classical Quantum Gravity & often & $\mathrm{ToC}$ & \\
\hline Cryo & Elsevier & Cryogenics & occasionally & $\mathrm{ToC}$ & \\
\hline CryRp & AIP & Crystallography Reports & occasionally & ToC & M \\
\hline CTM & IOP & Combustion Theory Modelling & often & $\mathrm{ToC}$ & \\
\hline DokPh & AIP & Physics - Doklady & occasionally & ToC & \\
\hline EJPh & IOP & European Journal of Physics & often & ToC & \\
\hline $\operatorname{InfPh}$ & Elsevier & Infrared Physics and Technology & often & abstracts & \\
\hline JAP & AIP & Journal of Applied Physics & often & $\mathrm{ToC}$ & M \\
\hline JATP & Elsevier & Journal Atmospheric and Terrestrial Physics & occasionally & ToC & \\
\hline $\mathrm{JChPh}$ & AIP & Journal of Chemical Physics & often & $\mathrm{ToC}$ & M \\
\hline $\mathrm{JCoPh}$ & $\mathrm{AP}$ & Journal of Computational Physics & occasionally & abstracts & \\
\hline JETP & AIP & JETP & occasionally & $\mathrm{ToC}$ & $\mathrm{M}$ \\
\hline JETPL & AIP & JETP Letters & occasionally & $\mathrm{ToC}$ & M \\
\hline JFS & $\mathrm{AP}$ & Journal of Fluids and Structures & occasionally & ToC & \\
\hline JGP & Elsevier & Journal of Geometry and Physics & occasionally & ToC & \\
\hline JLTP & OSA & Journal of Low Temperature Physics & occasionally & ToC & $\mathrm{M}$ \\
\hline JLwT & OSA & Journal of Lightwave Technology & occasionally & $\mathrm{ToC}$ & \\
\hline JMagR & $\mathrm{AP}$ & Journal of Magnetic Resonance & occasionally & abstracts & \\
\hline JMMM & Elsevier & Journal of Magnetism and Magnetic Materials & occasionally & abstracts & \\
\hline JMPS & AIP & Journal of Mathematical Physics & often & $\mathrm{ToC}$ & M \\
\hline JMoSp & $\mathrm{AP}$ & Journal of Molecular Spectroscopy & occasionally & abstracts & \\
\hline JNM & Elsevier & Journal of Nuclear Materials & occasionally & $\mathrm{ToC}$ & \\
\hline JPCM & IOP & Journal of the Physics of Condensed Matter & often & ToC & \\
\hline JPCRD & AIP & Journal of Physical and Chemical Reference Data & occasionally & ToC & M \\
\hline JPCS & Elsevier & Journal of Physics and Chemistry of Solids & occasionally & $\mathrm{ToC}$ & \\
\hline JPhA & IOP & Journal of Physics A: Mathematical General & often & ToC & \\
\hline $\mathrm{JPhB}$ & IOP & Journal of Physics B: Atomic Molecular Physics & often & $\mathrm{ToC}$ & \\
\hline JPhD & IOP & Journal of Physics D: Applied Physics & often & $\mathrm{ToC}$ & \\
\hline JPhG & IOP & Journal of Physics G: Nuclear Physics & often & $\mathrm{ToC}$ & \\
\hline JRheo & AIP & Journal of Rheology & often & ToC & M \\
\hline JSSCh & $\mathrm{AP}$ & Journal of Solid State Chemistry & occasionally & abstracts & \\
\hline JSV & $\mathrm{AP}$ & Journal of Sound and Vibration & often & ToC & \\
\hline $\mathrm{JTePh}$ & AIP & Journal of Technical Physics & occasionally & ToC & M \\
\hline MedPh & AIP & Medical Physics & often & $\mathrm{ToC}$ & M \\
\hline MSMSE & IOP & Modelling Simul. Mater. Sci. Eng. & often & ToC & \\
\hline MSSP & $\mathrm{AP}$ & Mechanical Systems \& Signal Processing & occasionally & abstracts & \\
\hline NIMPA & Elsevier & Nuclear Instruments/Methods Physics Research A & often & abstracts & \\
\hline NIMPB & Elsevier & Nuclear Instruments/Methods Physics Research B & often & abstracts & \\
\hline Nanot & IOP & Nanotechnology & often & $\mathrm{ToC}$ & \\
\hline NDS & $\mathrm{AP}$ & Nuclear Data Sheets & occasionally & abstracts & \\
\hline Nonli & IOP & Nonlinearity & often & $\mathrm{ToC}$ & \\
\hline NuGeo & Elsevier & Nuclear Geophysics & occasionally & ToC & \\
\hline $\mathrm{NuPhA}$ & Elsevier & Nuclear Physics A & weekly & abstracts & $\mathrm{E}$ \\
\hline $\mathrm{NuPhB}$ & Elsevier & Nuclear Physics B & weekly & abstracts & $\mathrm{E}$ \\
\hline $\mathrm{NuPhS}$ & Elsevier & Nuclear Physics B Proceedings Supplements & monthly & abstracts & $\mathrm{E}$ \\
\hline PAN & AIP & Physics of Atomic Nuclei & occasionally & $\mathrm{ToC}$ & $\mathrm{M}$ \\
\hline PCEB & Elsevier & Physics and Chemistry of the Earth Part B & occasionally & $\mathrm{ToC}$ & \\
\hline PCEC & Elsevier & Physics and Chemistry of the Earth Part C & occasionally & $\mathrm{ToC}$ & \\
\hline $\mathrm{PhFl}$ & AIP & Physics of Fluids & often & $\mathrm{ToC}$ & M \\
\hline PhLA & Elsevier & Physics Letters A & often & abstracts & \\
\hline PhLB & Elsevier & Physics Letters B & often & abstracts & \\
\hline
\end{tabular}


Table 4. continued

\begin{tabular}{|c|c|c|c|c|c|}
\hline Journal & Source & Full Name & How Often & Kind of Data & $\operatorname{Links}^{\mathrm{a}}$ \\
\hline $\mathrm{PhPl}$ & $\overline{\text { AIP }}$ & Physics of Plasmas & often & $\mathrm{ToC}$ & $\mathrm{M}$ \\
\hline $\mathrm{PhR}$ & Elsevier & Physics Reports & often & $\mathrm{ToC}$ & \\
\hline PhRvA & AIP & Physical Review A & often & $\mathrm{ToC}$ & M \\
\hline PhRvB & AIP & Physical Review B & often & $\mathrm{ToC}$ & M \\
\hline $\mathrm{PhRvC}$ & AIP & Physical Review C & often & $\mathrm{ToC}$ & M \\
\hline PhRvD & AIP & Physical Review D & often & $\mathrm{ToC}$ & M \\
\hline PhRvE & AIP & Physical Review E & often & $\mathrm{ToC}$ & M \\
\hline PhRvL & AIP & Physical Review Letters & often & $\mathrm{ToC}$ & M \\
\hline PhSS & AIP & Physics of the Solid State & occasionally & $\mathrm{ToC}$ & M \\
\hline $\mathrm{PhT}$ & AIP & Physics Today & occasionally & $\mathrm{ToC}$ & M \\
\hline PhyA & Elsevier & Physica A & often & $\mathrm{ToC}$ & \\
\hline PhyB & Elsevier & Physica B & often & abstracts & \\
\hline PhyC & Elsevier & Physica C & often & $\mathrm{ToC}$ & \\
\hline PhyD & Elsevier & Physica D & often & abstracts & \\
\hline PhyE & Elsevier & Physica E & occasionally & abstracts & \\
\hline PhyEd & IOP & Physics Education & often & $\mathrm{ToC}$ & \\
\hline PMB & IOP & Physics Medicine and Biology & often & $\mathrm{ToC}$ & \\
\hline $\mathrm{PPCF}$ & IOP & Plasma Physics and Controlled Fusion & often & $\mathrm{ToC}$ & \\
\hline $\mathrm{PPN}$ & AIP & Physics of Particles and Nuclei & occasionally & $\mathrm{ToC}$ & M \\
\hline PPNP & Elsevier & Progress in Particle and Nuclear Physics & occasionally & ToC & \\
\hline PQE & Elsevier & Progress in Quantum Electronics & occasionally & $\mathrm{ToC}$ & \\
\hline PSST & IOP & Plasma Sources Science Technology & often & ToC & \\
\hline QuSOp & IOP & Quantum Semiclassical Optics & often & $\mathrm{ToC}$ & \\
\hline $\mathrm{RaPC}$ & Elsevier & Radiation Physics and Chemistry & often & abstracts & \\
\hline $\mathrm{RPPh}$ & IOP & Reports on Progress in Physics & often & $\mathrm{ToC}$ & \\
\hline RvMP & AIP & Reviews of Modern Physics & occasionally & $\mathrm{ToC}$ & M \\
\hline Semic & AIP & Semiconductors & occasionally & $\mathrm{ToC}$ & M \\
\hline SeScT & IOP & Semiconductor Science Technology & often & $\mathrm{ToC}$ & \\
\hline SMaS & IOP & Smart Material Structures & often & $\mathrm{ToC}$ & \\
\hline SuScT & IOP & Superconductor Science Technology & often & $\mathrm{ToC}$ & \\
\hline SuMi & AP & Superlattices and Microstructures & occasionally & abstracts & \\
\hline TePhL & AIP & Technical Physics Letters & occasionally & $\mathrm{ToC}$ & M \\
\hline PhDT & UMI & University Microfilm, Inc. & occasionally & abstracts & \\
\hline WRM & IOP & Waves Random Media & often & $\mathrm{ToC}$ & \\
\hline
\end{tabular}

${ }^{\text {a }}$ Letter codes describing what data are available.

merging them into a single entry, avoiding duplication of redundant information.

\subsection{Tokenization}

The activity of parsing a (possibly) loosely-structured bibliographic record is typically more of an art than a science, given the wide range of possible formats used by people for the representation and display of these records. The ADS uses the PERL language (Practical Extraction and Report Language, Wall \& Schwartz 1991) for implementing most of the routines associated with handling the data. PERL is an interpreted programming language optimized for scanning and processing textual data. It was chosen over other programming languages because of its speed and flexibility in handling text strings. Features such as pattern matching and regular expression substitution greatly facilitate manipulating the data fields. To maximize flexibility in the parsing and formatting operations of different fields, we have written a set of PERL library modules and scripts capable of performing a few common tasks. Some that we consider worth mentioning from the methodological point of view are listed below.

- Character set conversion: electronic data are often delivered to us in different character set encodings, requiring translation of the data stream in one of the standard character sets expected by our input scripts. The default character set that has been used by the ADS until recently is "Latin-1" encoding (ISO-8859-1, International Organization for Standardization 1987). We are now in the process of converting to the use of Unicode characters (Unicode Consortium 1996) encoded in UTF-8 (UCS Transformation Format, 8-bit form). The advantage of using Unicode is its universality (all character sets can be mapped to Unicode without loss of information). The advantage of adopting UTF-8 over other encodings is mainly the software support currently available (most of the 
Table 5. Tagged format definitions

\begin{tabular}{|c|c|c|}
\hline Tag & Name & Comment \\
\hline$\% \mathrm{R}$ & Bibliographic Code & required \\
\hline$\% \mathrm{~T}$ & Title & required \\
\hline$\% \mathrm{~A}$ & Author List & required \\
\hline$\% \mathrm{D}$ & Publication Date & required \\
\hline$\% \mathrm{~B}$ & Abstract Text & \\
\hline$\% \mathrm{C}$ & Abstract Copyright & \\
\hline$\% \mathrm{E}$ & URL for Electronic Data Table & \\
\hline$\% \mathrm{~F}$ & Author Affiliation & \\
\hline$\% \mathrm{G}$ & Origin & \\
\hline$\% \mathrm{H}$ & Email & \\
\hline$\% \mathrm{~J}$ & Journal Name, Volume, and Page Range & \\
\hline$\% \mathrm{~K}$ & Keywords & \\
\hline$\% \mathrm{~L}$ & Last Page of Article & \\
\hline$\% \mathrm{O}$ & Object Name & \\
\hline$\% \mathrm{Q}$ & Category & \\
\hline$\% \mathrm{U}$ & URL for Electronic Document & \\
\hline$\% \mathrm{~V}$ & Language & \\
\hline$\% \mathrm{~W}$ & Database (AST, PHY, INST) & \\
\hline$\% \mathrm{X}$ & Comment & \\
\hline$\% \mathrm{Y}$ & Identifiers & \\
\hline$\% \mathrm{Z}$ & References & \\
\hline
\end{tabular}

modern software packages can already handle UTF-8 internally). The adoption of Unicode and UTF-8 also works well with our adoption of XML as the standard format for bibliographic data;

- Macro and entity expansion: Several of the highly structured document formats in use today rely on the strengths of the formatting language for the specification of some common formatting tasks or data tokens. Typically this means that LaTeX documents that are supplied to us make use of one or more macro packages to perform some of the formatting tasks. Similarly, SGML documents will conform to some Document Type Definition (DTD) provided to us by the publisher, and will make use of some standard set of SGML entities to encode the document at the required level of abstraction. What this means for us is that even if most of the input data comes to us in one of two basic formats (TeX/LaTeX/BibTeX or SGML/HTML/XML), we must be able to parse a large number of document classes, each one defined by a different and ever increasing set of specifications, be it a macro package or a DTD;

- Author name formatting: Special care has been taken in parsing and formatting author names from a variety of possible input formats to the standard one used by the ADS. The proper handling of author names is crucial to the integrity of the data in the ADS. Without proper author handling, users would be unable to get complete listings on searches by author names which comprise approximately two-thirds of all searches (see Eichhorn et al. 2000, hereafter SEARCH).
Since the majority of our data sources do not provide author names in our standard format (last name, first name or initial), our loading routines need to be able to invert author names accurately, handling cases such as multiple word last names (Da Costa, van der Bout, Little Marenin) and suffixes (Jr., Sr., III). Any titles in an author's name (Dr., Rev.) were previously omitted, but are now being retained in the new XML formatting of text files.

The assessment of what constitutes a multiple word last name as opposed to a middle name is non-trivial since some names, such as Davis, can be a first name (Davis Hartman), a middle name (A.G. Davis Philip), a last name (Robert Davis), or some combination (Davis S. Davis). Another example is how to determine when the name "Van" is a first name (Van Nguyen), a middle name (W. Van Dyke Dixon), or part of a last name (J. van Allen). Handling all of these cases correctly requires not only familiarity with naming conventions worldwide, but an intimate familiarity with the names of astronomers who publish in the field. We are continually amassing the latter as we incorporate increasing amounts of data into the system, and as we get feedback from our users;

- Spell checking: Since many of the historical records entered in the ADS have been generated by typesetting tables of contents, typographical errors can often be flagged in an automated way using spell-checking software. We have developed a PERL software driver for the international ispell program, a UNIX utility, which can be used as a spell-checking filter on all input to be considered textual information. A custom dictionary containing terms specific to astronomy and space sciences is used to increase the recognition capabilities of the software module. Any corrections suggested by the spell-checker module are reviewed by a human before the data are actually updated;

- Language recognition: Extending the capability of the spell-checker, we have implemented a software module which attempts to guess the language of an input text buffer based on the percentage of words that it can recognize in one of several languages: English, German, French, Spanish, or Italian. This module is used to flag records to be entered in our database in a language other than English. Knowledge of the language of an abstract allows us to create accurate synonyms for those words (see ARCHITECTURE).

\subsection{Identification}

We call identification the activity of mapping the tokens extracted from the parsing of a bibliographic record into a unique identifier. The ADS adopted the use of bibliographic codes as the identifier for bibliographic entries shortly after its inception, in order to facilitate communication between the ADS and SIMBAD. The advantage of 
using bibliographic codes as unique identifiers is that they can most often be created in a straightforward way from the information given in the list of references published in the astronomical literature, namely the publication year, journal name, volume, and page numbers, and first author's name (see Sect. 3.1 for details).

\subsection{Instantiation}

"Instantiation" of a bibliographic entry consists of the creation of a record for it in the ADS database. The ADS must handle receipt of the same data from multiple sources. We have created a hierarchy of data sources so that we always know the preferred data source. A reference for which we have received records from STI, the journal publisher, SIMBAD, and NED, for example, must be in the system only once with the best information from each source preserved. When we load a reference into the system, we check whether a text file already exists for that reference. If there is no text file, it is a new reference and a text file is created. If there already is a text file, we append the new information to the current text file, creating a "merged" text file. This merged text file lists every instance of every field that we have received.

\subsection{Extraction}

By "extraction" of a bibliographic entry we mean the procedure used to create a unique representation of the bibliography from the available records. This is essentially an activity of data fusion and unification, which removes redundancies in the bibliographic records obtained by the ADS and properly labels fields by their characteristics. The extraction algorithm has been designed with our prior experience as to the quality of the data to select the best fields from each data source, to cross-correlate the fields as necessary, and to create a "canonical" text file which contains a unique instance of each field. Since the latter is created through software, only one version of the text file must be maintained; when the merged text file is appended, the canonical text file is automatically recreated.

The extraction routine selects the best pieces of information from each source and combines them into one reference which is more complete than the individual references. For example, author lists received from STI were often truncated after five or ten authors. Whenever we have a longer author list from another source, that author list is used instead. This not only recaptures missing authors, it also provides full author names instead of author initials whenever possible. In addition, our journal sources sometimes omit the last page number of the reference, but SIMBAD usually includes it, so we are able to preserve this information in our canonical text file.

Some fields need to be labelled by their characteristics so that they are properly indexed and displayed. The keywords, for example, need to be attributed to a specific keyword system. The system designation allows for multiple keyword sets to be displayed (e.g. NASA/STI Keywords and AAS Keywords) and will be used in the keyword synonym table currently under development (Lee et al. 1999).

We also attempt to cross-correlate authors with their affiliations wherever possible. This is necessary for records where the preferred author field is from one source and the affiliations are from another source. We attempt to assign the proper affiliation based on the last name and do not assume that the author order is accurate since we are aware of ordering discrepancies in some of the STI records.

Through these four steps in the procedure of creating and managing bibliographic records, we are able to take advantage of receiving the same reference from multiple sources. We standardize the various records and present to the user a combination of the most reliable fields from each data source in one succinct text file.

\section{Updating the database}

The software to update bibliographic records in the database consists of a series of PERL scripts, typically one per data source, which reads in the data, performs any special processing particular to that data source, and writes out the data to text files. The loading routines perform three fundamental tasks: 1) they add new bibliographic codes to the current master list of bibliographic codes in the system; 2) they create and organize the text files containing the reference data; and 3) they maintain the lists of bibliographic codes used to indicate what items are available for a given reference.

\subsection{The master list}

The master list is a table containing bibliographic codes together with their publication dates (YYYYMM) and entry dates into the system (YYYYMMDD). There is one master list per database with one line per reference. The most important aspect of the master list is that it retains information about "alternative" bibliographic codes and matches them to their corresponding preferred bibliographic code. An alternative bibliographic code is usually a reference which we receive from another source (primarily SIMBAD or NED) which has been assigned a different bibliographic code from the one used by the ADS. Sometimes this is due to the different rules used to build bibliographic codes for non-standard publications (see Sect. 3.1), but often it is just an incorrect year, volume, page, or author initial in one of the databases (SIMBAD or NED or the ADS). In either case, the ADS must keep the alternative bibliographic code in the system so that it can be found when referenced by the other source (e.g. when 
SIMBAD sends back a list of their codes related to an object). The ADS matches the alternative bibliographic code to our corresponding one and replaces any instances of the alternative code when referenced by the other data source. Alternative bibliographic codes in the master list are prepended with an identification letter ( $\mathrm{S}$ for SIMBAD, N for NED, J for Journal) so that their origin is retained.

While we make every effort to propagate corrections back to our data sources, sometimes there is simply a valid discrepancy. For example, alternative bibliographic codes are often different from the ADS bibliographic code due to ambiguous differences such as which name is the surname of a Chinese author. Since Americans tend to invert Chinese names one way (Zheng, Wei) and Europeans another (Wei, Zheng), this results in two different, but equally valid codes. Similarly, discrepancies in journal names such as BAAS (for the published abstracts in the Bulletin of the American Astronomical Society) and AAS (for the equivalent abstract with meeting and session number, but no volume or page number) need different codes to refer to the same paper. Russian and Chinese translation journals (Astronomicheskii Zhurnal vs. Soviet Astronomy and Acta Astronomica Sinica vs. Chinese Astronomy and Astrophysics) share the same problem. These papers appear once in the foreign journal and once in the translation journal (usually with different page numbers), but are actually the same paper which should be in the system only once. The ADS must therefore maintain multiple bibliographic codes for the same article since each journal has its own abbreviation, and queries for either one must be able to be recognized. The master list is the source of this correlation and enables the indexing procedures and search engine to recognize alternative bibliographic codes.

\subsection{The text files}

Text files in the ADS are stored in a directory tree by bibliographic code. The top level of directories is divided into directories with four-digit names by publication year (characters 1 through 4 of the bibliographic code). The next level contains directories with five-character names according to journal (characters 5 through 9), and the text files are named by full bibliographic code under these journal directories. Thus, a sample pathname is 1998/MNRAS/1998MNRAS.295...75E. Alternative bibliographic codes do not have a text file named by that code, since the translation to the equivalent preferred bibliographic code is done prior to accessing the text file.

A sample text file is given in the appendices. Appendix B shows the full bibliographic entry, including all records as received from STI, MNRAS, and SIMBAD. It contains XML-tagged fields from each source, showing all instances of every field. Appendix $\mathrm{C}$ shows the extracted canonical version of the bibliographic entry which contains only selected information from the merged text file. This latter version is displayed to the user through the user interface (see SEARCH).

\subsection{The codes files}

The third basic function of the loading procedures is to modify and maintain the listings for available items. The ADS displays the availability of resources or information related to bibliographic entries as letter codes in the results list of queries and as more descriptive hyperlinks in the page displaying the full information available for a bibliographic entry. A full listing of the available item codes and their meaning is given in SEARCH.

The loading routines maintain lists of bibliographic codes for each letter code in the system which are converted to URLs by the indexing routines (see ARCHITECTURE). Bibliographic codes are appended to the lists either during the loading process or as post-processing work depending on the availability of the resource. When electronic availability of data coincides with our receipt of the data, the bibliographic codes can be appended to the lists by the loading procedures. When we receive the data prior to electronic availability, post-processing routines must be run to update the bibliographic code lists after we are notified that we may activate the links.

\section{The articles}

The ADS is able to scan and provide free access to past issues of the astronomical journals because of the willing collaboration of the journal publishers. The primary reason that the journal publishers have agreed to allow the scanning of their old volumes is that the loss of individual subscriptions does not pose a threat to their livelihood. Unlike many disciplines, most astronomy journals are able to pay for their publications through the cost of page charges to astronomers who write the articles and through library subscriptions which are unlikely to be cancelled in spite of free access to older volumes through the ADS. The journal publishers continue to charge for access to the current volumes, which is paid for by most institutional libraries. This arrangement places astronomers in a fortunate position for electronic accessibility of astronomy articles.

The original electronic publishing plans for the astronomical community called for STELAR (STudy of Electronic Literature for Astronomical Research, van Steenberg 1992; van Steenberg et al. 1992; Warnock et al. 1992; Warnock et al. 1993) to handle the scanning and dissemination of the full journal articles. However, when the STELAR project was terminated in 1993, the ADS assumed responsibility for providing 
scanned full journal articles to the astronomical community. The first test journal to be scanned was the ApJ Letters which was scanned in January, 1995 at 300 dots per inch (dpi). It should be noted that those scans were intended to be $600 \mathrm{dpi}$ and we will soon rescan them at the higher $600 \mathrm{dpi}$ resolution. Complications in the journal publishing format (plates at the end of some volumes and in the middle of others) were noted and detailed instructions provided to the scanning company so that the resulting scans would be named properly by page or plate number.

All of the scans since the original test batch have been scanned at 600 dpi using a high speed scanner and generating a 1 bit/pixel monochrome image for each page. The files created are then automatically processed in order to de-skew and center the text in each page, resize images to a standard U.S. Letter size $(8.5 \times 11$ inches $)$, and add a copyright notice at the bottom of each page. For each original scanned page, two separate image files of different resolutions are generated and stored on disk. The availability of different resolutions allows users the flexibility of downloading either high or medium quality documents, depending on the speed of their internet connection. The image formats and compression used were chosen based on the available compression algorithms and browser capabilities. The high resolution files currently used are 600 dpi, 1 bit/pixel TIFF (Tagged Image File Format) files, compressed using the CCITT Group 4 facsimile encoding algorithm. The medium resolution files are $200 \mathrm{dpi}$, $1 \mathrm{bit} /$ pixel TIFF files, also with CCITT Group 4 facsimile compression.

Conversion to printing formats (PDF, PCL, and Postscript) is done on demand, as requested by the user. Similarly, conversion from the TIFF files to a low resolution GIF (Graphic Interchange Format) file (75, 100, or $150 \mathrm{dpi}$, depending on user preferences) for viewing on the computer screen is done on demand, then cached so that the most frequently accessed pages do not need to be created every time. A procedure run nightly deletes the GIF files with the oldest access time stamp so that the total size of the disk cache is kept under a pre-defined limit. The current 10 GBytes of cache size in use at the SAO Article Server causes only files which have not been accessed for about a month to be deleted. Like the full-screen GIF images, the ADS also caches thumbnail images of the article pages which provide users with the capability of viewing the entire article at a glance.

The ADS uses Optical Character Recognition (OCR) software to gain additional data from TIFF files of article scans. The OCR software is not yet adequate for accurate reproduction of the scanned pages. Greek symbols, equations, charts, and tables do not translate accurately enough to remain true to the original printed page. For this reason, we have chosen not to display to the user anything rendered by the OCR software in an unsuper- vised fashion. However, we are still able to take advantage of the OCR software for several purposes.

First, we are able to identify and extract the abstract paragraph(s) for use when we do not have the abstract from another source. In these cases, the OCR'd text is indexed so that it is searchable and the extracted image of the abstract paragraph is displayed in lieu of an ASCII version of the abstract. Extracting the abstract from the scanned pages is somewhat tedious, as it requires establishing different sets of parameters for each journal, as well as for different fonts used over the years by the same journal. The OCR software can be taught how to determine where the abstract ends, but it does not work for every article due to oddities such as author lists which extend beyond the first page of an article, and articles which are in a different format from others in the same volume (e.g. no keywords or multiple columns). The ADS currently contains approximately 25000 of these abstract images and more will be added as we continue to scan the historical literature.

We are also currently using the OCR software to render electronic versions of the entire scanned articles for indexing purposes. We will not use this for display to the users, but hope to be able to index it to provide the possibility of full text searching at some future date. We estimate that the indexing of our almost one million scanned pages with our current hardware and software will take approximately two years of dedicated CPU time.

The last benefit that we gain from the OCR software is the conversion of the reference list at the end of articles. We use parsed reference lists from the scanned articles to build citation and reference lists for display through the $\mathrm{C}$ and $\mathrm{R}$ links of the available items. Since reference lists are typically in one of several standard formats, we parse each reference for author, journal, volume and page number for most journal articles, and conference name, author, and page number for many conference proceedings. This enables us to build bibliographic code lists for references contained in that article ( $\mathrm{R}$ links) and invert these lists to build bibliographic code lists of articles which cite this paper ( $\mathrm{C}$ links). We are able to use this process to identify and therefore add commonly-cited articles which are currently missing from the ADS. This is usually data prior to 1975 or astronomy-related articles published in non-astronomy journals.

The Article Service currently contains 250 GBytes of scans, which consists of 1128955 article pages comprising 138789 articles. These numbers increase on a regular basis, both as we add more articles from the older literature and as we scan new journals.

\section{ADS/Journal interaction}

A description of the data in the ADS would be incomplete without a discussion of the interaction between the 
ADS and the electronic journals. The data available online from the journal publishers is an extension of the data in the ADS and vice versa. This interaction is greatly facilitated by the acceptance of the bibliographic code by many journal publishers as a means for accessing their on-line articles.

Access to articles currently on-line at the journal sites through the ADS comprises a significant percent of the online journal access (see OVERVIEW). The best model for interaction between the ADS and a journal publisher is the University of Chicago Press (hereafter UCP), publisher of $A p J, A p J L, A p J S, A J$, and PASP. When a new volume appears on-line at UCP, the ADS is notified by email and an SGML header file for each of those articles is simultaneously transferred to our site. The data are parsed and loaded into the system and appropriate links are created. However, prior to this, the UCP has made use of the ADS to build their electronic version through the use of our bibliographic code reference resolver.

Our bibliographic code reference resolver (Accomazzi et al. 1999) was developed to provide the capability to automatically parse, identify, and verify citations appearing in astronomical literature. By verifying the existence of a reference through the ADS, journals and conference proceedings editors are able to publish documents containing hyperlinks pointing to stable, unique URLs. Increasingly more journals are linking to the ADS in their reference sections, providing users with the ability to read referenced articles with the click of a mouse button.

During the copy editing phase, UCP editors query the ADS reference resolver and determine if each reference exactly matches a bibliographic code in the ADS. If there is a match, a link to the ADS is established for this entry in their reference section. If there is not a match, one of several scenarios takes place. First, if it is a valid reference not yet included in the ADS (most often the case for "fringe" articles, those peripherally associated with astronomy), our reference resolver captures the information necessary to add it to our database during the next update. Second, if it is a valid reference unable to be parsed by the resolver (sometimes the case for conference proceedings or PhD theses), no action is taken and no link is listed in the reference section. Third, if there is an error in the reference as determined by the reference resolver, the UCP editors may ask for a correction or clarification from the authors.

The last option demonstrates the power of the reference resolver, which has been taught on a journal-byjournal basis how complete the coverage of that journal is in the ADS. Before the implementation of the reference resolver, UCP was able to match $72 \%$ of references in $A p J$ articles (E. Owens, private communication). Early results from the use of the reference resolver show that we are now able to match conference proceedings, so this number should become somewhat larger. It is unlikely that we will ever match more than $90 \%$ of references in an article due to references such as "private communication", "in press", and preprints, as well as author errors (see Sect. 8). Our own reference resolving of OCR'd reference lists shows that we can match approximately $86 \%$ of references for the best-case scenario.

The ADS provides multiple ways for authors and journal publishers to link to the ADS (see SEARCH). We make every effort to facilitate individuals and organizations linking to us. This is easily done for simple searches such as the verification of a bibliographic code or an author search for a single spelling. However, given the complexity of the system, these automated searches can quickly become complicated. Details for conference proceedings editors or journal publishers who are interested in establishing or improving links to the ADS are available upon request. In particular, those who have individual TeX macros incorporated in their references can use our bibliographic code resolver to facilitate linking to the ADS.

\section{Discussion and summary}

As of this writing (12/1999), there are 524304 references in the Astronomy database, 523498 references in the Instrumentation database, 443858 references in the Physics database, and 3467 references in the Preprint database, for a total of almost 1.5 million references in the system. Astronomers currently write approximately 18000 journal articles annually, and possibly that many additional conference proceedings papers per year. More than half of the journal papers appear in peer-reviewed journals. These numbers are more than double what they were in 1975, in spite of an increase in the number of words per page in most of the major journals (Abt 1995), and an increase in number of pages per article (Schulman et al. 1997). At the current rate of publication, astronomers could be writing 25000 journal papers per year by 2001 and an additional 20000 conference proceedings papers. Figure 1 shows the total number of papers for each year in the Astronomy database since 1975, divided into refereed journal papers, non-refereed journal papers, and conferences (including reports and theses). There are three features worth noting. First, the increase in total references in 1980 is due to the inclusion of Helen Knudsen's Monthly Astronomy and Astrophysics Index, a rich source of data for both journals and conference proceedings which began coverage in late 1979 and continued until 1995. Second, the recent increase in conferences included in the Astronomy database (starting around 1996) is due to the inclusion of conference proceedings table of contents provided by collaborating librarians and typed in by our contractors. Last, the decrease in numbers for 1999 is due to coverage for that year not yet being complete in the ADS.

The growth rate of the Instrumentation and Physics databases is difficult to estimate, primarily because we do 


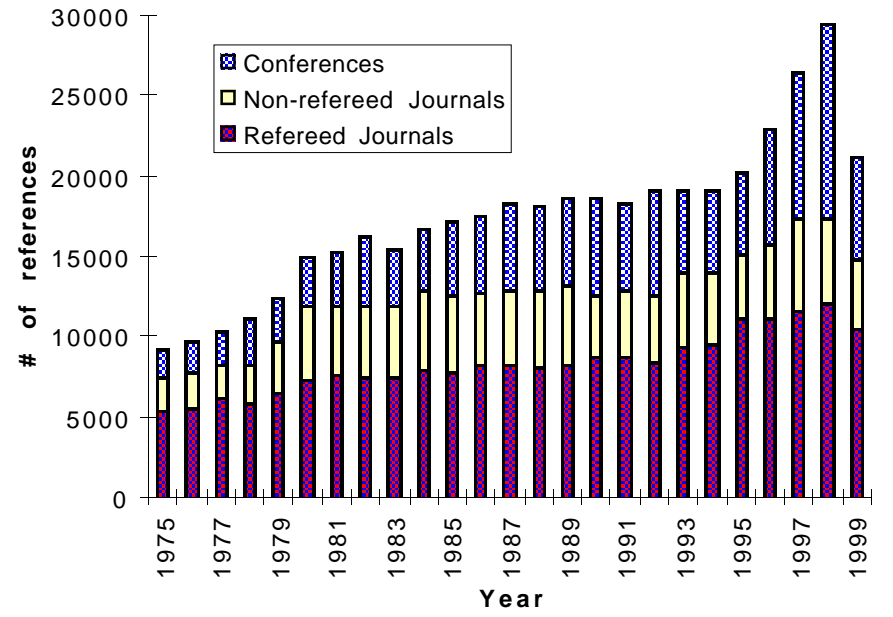

Fig. 1. Histogram showing the number of refereed journal papers, non-refereed journal papers, and conferences (including reports and theses) for each year in the Astronomy database since 1975

not have datasets which are as complete as astronomy. In any case, the need for the organization and maintenance of this large volume of data is clearly important to every research astronomer. Fortunately, the ADS was designed to be able to handle this large quantity of data and to be able to grow with new kinds of data. New available item links have been added for new types of data as they became available (e.g. the links to complete book entries at the Library of Congress) and future datasets (e.g. from future space missions) should be able to be added in the same fashion.

As with any dataset of this magnitude, there is some fraction of references in the system which are incorrect. This is unavoidable given the large number of data sources, errors in indices and tables of contents as originally published, and human error. In addition, many authors do not give full attention to verifying all references in a paper, resulting in the introduction of errors in many places. In a systematic study of more than 1000 references contained in a single issue of the Astrophysical Journal, Abt (1992) found that more than $12 \%$ of those contained errors. This number should be significantly reduced with the integration of the ADS reference resolver in the electronic publishing process. However, any mistakes in the ADS can and will get propagated, so steps are being taken by us to maximize accuracy of our entries.

Locating and identifying correlations between multiple bibliographic codes which describe the same article is a time-consuming and sometimes subjective task as many pairs of bibliographic codes need to be verified by manually looking up papers in the library. We use the Abstract Service itself for gross matching of bibliographic codes, submitting a search with author and title, and considering any resulting matches with a score of 1.0 as a potential match. These matches are only potential matches which require verification since authors can submit the same paper to more than one publication source (e.g. BAAS and a refereed journal), and since errata published with the same title and author list will perfectly match the original paper.

When a volume or year is mismatched, it is usually obvious which of a pair of matched bibliographic codes is correct, but if a page number is off, the decision as to which code is correct cannot always be automated. We also need to consider matches with very high scores less than 1.0 since these are the matches where an author name may be incorrect. The correction of errors of this sort is ongoing work which is carried out as often as time and resources permit.

The evolution of the Internet and the World Wide Web, along with the explosion of astronomical services on the Web has enabled the ADS to provide access to our databases in an open and uniform environment. We have been able to hyperlink both to our own resources and to other on-line resources such as the journal bibliographies (Boyce \& Biemesderfer 1996). As part of the international collaboration Urania (Universal Research Archive of Networked Information in Astronomy, Boyce 1998), the ADS enables a fully functioning distributed digital library of astronomical information which provides power and utility previously unavailable to the researcher.

Perhaps the largest factor which has contributed to the success of the ADS is the willing cooperation of the AAS, CDS, and all the journal publishers. The ADS has largely become the means for linking together smaller pieces of a bigger picture, making an elaborate digital library for astronomers a reality. We currently collaborate with over fifty groups in creating and maintaining cross-links among data centers. These additional collaborations with individuals and institutions worldwide allow us to provide many value-added features to the system such as object information, author email addresses, mail order forms for articles, citations, article scans, and more. A listing of these collaborations is provided in Table 6. Any omissions from this table are purely unintentional, as the ADS values all of our colleagues and the users benefit not only from the major collaborators but the minor ones as well, as these are often more difficult for users to learn about independently. Most of the abbreviations are listed in Tables 2, 3, and 4 .

The successful coordination of data exchanges with each of our collaborators and the efforts which went into establishing them in the first place have been key to the success of the ADS. Establishing links to and from the journal publishers, changing these links due to revisions at publisher websites, and tracking and fixing broken links is all considered routine data maintenance for the system. Since it is necessary for us to maintain connectivity to external sites, routine checks of sample links are performed on a regular basis to verify that the links are still active. 
Table 6. Collaborators

\begin{tabular}{|c|c|}
\hline Additional Collaborations & Nature of the Collaboration \\
\hline A.G. Davis Philip & Scanning of Conference Proceedings \\
\hline Academic Press (AP) & Scanning of Icarus \\
\hline American Astronomical Society (AAS) & Citations, Scanning of AJ, ApJ, ApJL, ApJS, AASPB ${ }^{a}$, BAAS \\
\hline American Institute of Physics & Scanning of SvAL \\
\hline Andre Heck & Star Heads (Author Home Pages) \\
\hline Annual Reviews, Inc. & Scanning of ARA\&A \\
\hline Astronomical Data Center (ADC) & D links to data \\
\hline Astronomical Institute of Czechoslovakia & Scanning of BAICz \\
\hline Astronomical Institute of the Slovak Academy of Sciences & Scanning of CoSka \\
\hline Astronomical Society of Australia & Scanning of PASA \\
\hline Astronomical Society of India & Scanning of BASI \\
\hline Astronomical Society of Japan & Scanning of PASJ \\
\hline Astronomical Society of the Pacific (ASP) & Scanning of PASP and Conference Proceedings \\
\hline Astronomische Gesellschaft & Scanning of RvMA \\
\hline Astronomische Nachrichten & Scanning of AN \\
\hline Baltic Astronomy & Scanning of BaltA \\
\hline British Astronomical Association & Scanning of JBAA \\
\hline Cambridge University Press & M links to order forms, Scanning \\
\hline Central Bureau for Astronomical Telegrams (CBAT) & Object searches \\
\hline Chris Benn & Astropersons.lis (Author Email) \\
\hline EDP Sciences & Scanning of A\&AS \\
\hline Elsevier Publishers & E links to articles \\
\hline General Catalogue of Photometric Data (GCPD) & D links to data \\
\hline Institute for Scientific Information (ISI) & Citations \\
\hline International Society for Optical Engineering (SPIE) & M links to order forms \\
\hline Korean Astronomical Society & Scanning of JKAS \\
\hline Kluwer Publishers & M links to order forms, Scanning of SoPh \\
\hline Library of Congress (LOC) & Z39.50 interface, L links to data \\
\hline Los Alamos National Laboratory (LANL) & Preprint Archive \\
\hline Lunar and Planetary Science Institute (LPI) & Scanning, Object searches \\
\hline Meteoritical Society & Scanning of M\&PS \\
\hline NED & $\mathrm{N}$ links to objects, Object searches \\
\hline The Observatory & Scanning \\
\hline Royal Astronomical Society & Scanning of MNRAS \\
\hline SIMBAD & S links to objects, D links to data, Object searches \\
\hline Springer Verlag & Scanning of $\mathrm{A} \& \mathrm{~A}, \mathrm{ZA}^{\mathrm{b}}$ \\
\hline Universitad Nacional Autonoma de Mexico (UNAM) & Scanning of RMxAA, RMxAC \\
\hline University of Chicago Press (UCP) & Reference Resolving \\
\hline
\end{tabular}

a American Astronomical Society Photo Bulletin.

b Zeitschrift für Astrophysik.

Usage statistics for the Abstract Service (see OVERVIEW) indicate that astronomers and librarians at scientific institutions are eager to take advantage of the information that the ADS provides. The widespread acceptance of the ADS by the astronomical community is changing how astronomers do research, placing extensive bibliographic information at their fingertips. This enables researchers to increase their productivity and to improve the quality of their work.

A number of improvements to the data in the ADS are planned for the near future. As always, we will continue our efforts to increase the completeness of coverage, particularly for the data prior to 1975 . We have collected most of the major journals back to the first issue for scanning and adding to the Astronomy database. In addition, we are scanning and OCR'ing table of contents for conference proceedings to improve our coverage in that area. We are currently OCR'ing full journal articles to provide full text searching and to improve the completeness of our reference and citation coverage. Finally, as the ADS becomes commonplace for all astronomers, valuable feedback from our users to inform us about missing papers, errors in the database, and suggested improvements to the system serve to guide the future of the ADS and to ensure that the ADS continues to evolve into a more valuable research tool for the scientific community. 
Acknowledgements. The other ADS Team members: Markus Demleitner, Elizabeth Bohlen, and Donna Thompson contribute much on a daily basis. Funding for this project has been provided by NASA under NASA Grant NCC5-189.

\section{Appendix A}

Version 1.0 of the XML DTD describing text files in the ADS Abstract Service.

Document Type Definition for the ADS

bibliographic records

Syntax policy

- The element names are in uppercase in order to help the reading.

- The attribute names are preferably in lowercase

- The attribute values are allowed to be of type CDATA to allow more flexibility for additional values; however, attributes typically may only assume one of a welldefined set of values

- Cross-referencing among elements such as $\mathrm{AU}, \mathrm{AF}$, and $\mathrm{EM}$ is accomplished through the use of attributes of type IDREFS (for AU) and ID (for AF and EM)

$<$ !-- BIBRECORD is the root element of the XML document. Attributes are:

origin mnemonic indicating individual(s) or institution(s) who submitted the record to ADS

lang language in which the contents of this record are expressed the possible values are language tags as defined in RFC 1766.

Examples: lang="fr", lang="en"

$-->$

<!ELEMENT BIBRECORD ( METADATA?,

TITLE?,

AUTHORS?,

AFFILIATIONS?,

EMAILS?,

FOOTNOTES?,

BIBCODE,

MSTRING,

MONOGRAPH? ,

SERIES?,

PAGE?,

LPAGE?, COPYRIGHT?,

PUBDATE,

CATEGORIES*,

COMMENTS*,

ANOTE?,

BIBTYPE?,

\author{
IDENTIFIERS?, \\ ORIGINS, \\ OBJECTS*, \\ KEYWORDS*, \\ ABSTRACT* ) > \\ $<$ !ATTLIST BIBRECORD origin CDATA \#REQUIRED \\ lang CDATA \#IMPLIED > \\ <!-- Generic metadata about the ADS record \\ (rather than the publication) --> \\ $<$ ! ELEMENT METADATA ( VERSION, \\ CREATOR, \\ CDATE, \\ EDATE ) >
}

<!-- Versioning is introduced to allow parsers to detect and reject any documents not complying with the supported DTD $-->$ $<$ !ELEMENT VERSION ( \#PCDATA) >

$<$ !-- CREATOR is purely informative --> $<$ !ELEMENT CREATOR ( \#PCDATA) >

$<$ !-- Creation date for the record --> $<$ !ELEMENT CDATE ( YYYY-MM-DD) >

$<$ !-- Last modified date -->

$<$ !ELEMENT EDATE ( YYYY-MM-DD ) >

$<$ !-- Title of the publication -->

$<$ !ELEMENT TITLE ( \#PCDATA ) >

$<$ !ATTLIST TITLE lang CDATA \#IMPLIED >

<!-- AUTHORS contains only AU subelements, each one of them corresponding to a single author name $-\rightarrow$

$<$ !ELEMENT AUTHORS $(\mathrm{AU}+)>$

<!-- AU contains at least the person's last name (LNAME), and possibly the first and middle name(s) (or just the initials) which would be stored in element FNAME. PREF and SUFF represent the salutation and suffix for the name. SUFF typically is one of: Jr., Sr., II, III, IV. PREF is rarely used but is here for completeness. Typically we would store salutations such as "Rev." (for "Reverend"), or "Prof." (for "Professor") in this element.

$-->$

$<$ !ELEMENT AU ( PREF?, FNAME?, LNAME,

SUFF? ) >

<!-- The attributes AF and EM are used to crossreference author affiliations and email addresses with the individual author records. This is the only exception of attributes in upper case. The typical use of this is: $\langle\mathrm{AU}$ AF="AF_1 AF_2" EM="EM_3" $\rangle ..</ A U\rangle$

$-->$

$<$ !ATTLIST AU AF IDREFS \#IMPLIED 


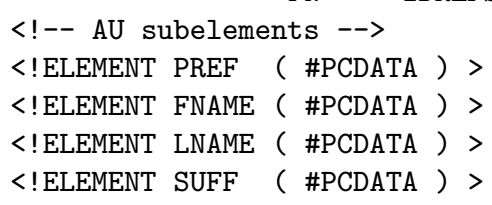

$<$ !-- FOOTNOTES and FN subelements are here for future use -->

$<$ !ELEMENT FOOTNOTES $(\mathrm{FN}+)>$

$<$ !ELEMENT FN ( \#PCDATA ) >

$<$ IATTLIST FN ident ID \#REQUIRED >

<!-- BIBCODE; for a definition, see:

http://adsdoc.harvard.edu/abs_doc/bib_help.html

http://adsabs.harvard.edu/cgi-bin/ nph-bib_query?1995ioda.book. .259S

http://adsabs .harvard.edu/cgi-bin/ nph-bib_query?1995VA.....39R.272S

This identifier logically belongs to the IDENTS element, but since it is the identifier used internally in the system, it is important to have it in a prominent and easy to reach place.

$-->$

$<$ !ELEMENT BIBCODE ( \#PCDATA ) >

$<$ !-- MSTRING is the unformatted string for the monograph (article, book, whatever). Example: <MSTRING>The Astrophysical Journal, Vol. 526, n. 2, pp. L89-L92</MSTRING>

$-->$

$<$ !ELEMENT MSTRING ( \#PCDATA) >

$<$ !-- MONOGRAPH is a structured record containing the fielded information about the monograph where the bibliographic entry appeared. Typically this is created by parsing the text in the MSTRING element. Example: <MTITLE>The Astrophysical Journal</MTITLE> $<$ VOLUME $>526</$ VOLUME $>$ $<$ ISSUE $>2</$ ISSUE $>$

$<$ PUBLISHER>University of Chicago Press $</$ PUBLISHER $>$
$-->$

$<$ !ELEMENT MONOGRAPH ( MTITLE,

VOLUME?,

ISSUE?,

MNOTE?,

EDITORS?,

EDITION?, PUBLISHER?, LOCATION?, MID* ) >

<!-- Monograph title (e.g. "Astrophysical Journal") $-\rightarrow$

$<$ !ELEMENT MTITLE ( \#PCDATA ) >

$<$ !ELEMENT VOLUME ( \#PCDATA) >

$<$ !ATTLIST VOLUME type NMTOKEN \#IMPLIED >

$<$ !ELEMENT ISSUE ( \#PCDATA ) >

$<$ !-- A note about the monograph as supplied by the publisher or editor -->

$<$ !ELEMENT MNOTE ( \#PCDATA) >

$<$ !-- List of editor names as extracted from MSTRING.

Formatting is as for AUTHORS and AU elements -->

$<$ !ELEMENT EDITORS $(\mathrm{ED}+)>$

$<$ !ELEMENT ED ( PREF?,

FNAME?,

LNAME,

SUFF? ) >

$<$ !-- Edition of publication -->

$<$ !ELEMENT EDITION ( \#PCDATA ) >

$<$ !-- Name of publisher -->

$<$ ! ELEMENT PUBLISHER ( \#PCDATA) >

$<$ !-- Place of publication -->

$<$ !ELEMENT LOCATION ( \#PCDATA) >

$<$ !-- MID represents the monograph identification as supplied by the publisher. This may be useful in correlating our record with the publisher's online offerings. The "system" attribute characterizes the system used to express the identifier -->

$<$ !ELEMENT MID ( \#PCDATA ) >

$<$ !ATTLIST MID type NMTOKEN \#IMPLIED >

<!-- If the bibliographic entry appeared in a series, then the element SERIES contains information about the series itself. Typically this consists of data about a conference series (e.g. ASP Conference Series). Note that there may be several SERIES elements, since some publications belong to "subseries" within a series.

$-->$

$<$ !ELEMENT SERIES ( SERTITLE, SERVOL? SEREDITORS?, SERBIBCODE? ) >

$<$ !-- Title, volume, and editors of conference series -->

$<$ !ELEMENT SERTITLE ( \#PCDATA ) >

$<$ !ELEMENT SERVOL ( \#PCDATA ) >

$<$ !ELEMENT SEREDITORS ( ED+ ) >

$<$ !-- Serial bibcode for publication (may coincide 
with main bibcode) -->

$<$ !ELEMENT SERBIBCODE ( \#PCDATA ) >

<!-- PAGE may have the attribute type set to "s" for (sequential) the value associated to it does not represent a printed volume number $-\rightarrow$

$<$ !ELEMENT PAGE ( \#PCDATA ) >

$<$ !ATTLIST PAGE type NMTOKEN \#IMPLIED >

<!-- LPAGE gives the last page number (if known). Does not make sense if PAGE is type="s" --> $<$ !ELEMENT LPAGE ( \#PCDATA) >

<!-- COPYRIGHT is just an unformatted string containing copyright information from publisher $-\rightarrow>$

$<$ !ELEMENT COPYRIGHT ( \#PCDATA ) >

$<$ !ELEMENT PUBDATE ( YEAR, MONTH? ) >

$<$ ! ELEMENT MONTH ( \#PCDATA ) >

$<$ !ELEMENT YEAR ( \#PCDATA ) >

<!-- CATEGORIES contain subelements indicating in which subject categories the publication was assigned. STI/RECON has always assigned a category for each entry in their system, but otherwise there is little else in our database. The attributes origin and system are used to keep track of the different classifications used.

$-->$

$<$ !ELEMENT CATEGORIES $(\mathrm{CA}+)>$

$<$ !ATTLIST CATEGORIES origin NMTOKEN \#IMPLIED system NMTOKEN \#IMPLIED >

$<$ !ELEMENT CA ( \#PCDATA ) >

<!-- Typically private fields supplied by the data source. For instance, SIMBAD and LOC provide comments about a bibliographic entries -->

$<$ !ELEMENT COMMENTS $(\mathrm{CO}+)>$

$<$ !ATTLIST COMMENTS lang CDATA \#IMPLIED origin NMTOKEN \#IMPLIED >

$<$ !ELEMENT CO ( \#PCDATA ) >

$<$ !-- Author note -->

$<$ !ELEMENT ANOTE ( \#PCDATA) >

<!-- BIBTYPE describes what type of publication this entry corresponds to. This is currently limited to the following tokens (taken straight from the BibTeX classification):

article

book

booklet

inbook

incollection

inproceedings

manual

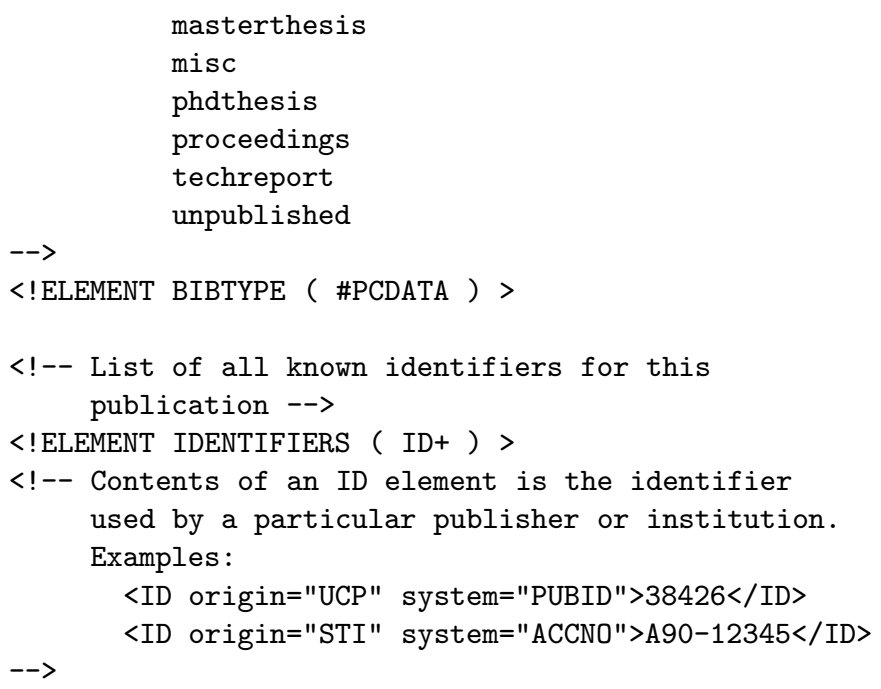

<!-- An abstract of the publication. This is typically provided to us by the publisher, but may in some cases come from other sources (E.g. STI, which keyed abstracts in most cases). Therefore we allow several ABSTRACT elements within each record, each with a separate origin or language. The attribute type is used to keep track of how the abstract data was generated. For instance, abstract text generated by our OCR software will have:

$-->$ origin="ADS" type="OCR" lang="en"

$<$ !ELEMENT ABSTRACT $(\mathrm{P}+)>$

$<$ !ATTLIST ABSTRACT origin NMTOKEN \#IMPLIED > type NMTOKEN \#IMPLIED > lang CDATA \#IMPLIED >

<!-- Abstracts are composed of separate paragraphs which have mixed contents as listed below. All the subelements listed below have the familiar HTML meaning and 
are used to render the abstract text in a decent way $-->$

$<$ !ELEMENT P (\#PCDATA |A| BR | PRE | SUP | SUB)* >

$<$ !-- Line breaks (BR) and preformatted text (PRE) make it possible to display tables and other preformatted text. -->

$<$ !ELEMENT BR EMPTY >

$<$ !ELEMENT PRE (\#PCDATA | A | BR | SUP | SUB )* >

$<$ !-- A is the familiar anchor element. -->

$<$ !ELEMENT A (\#PCDATA | BR | SUP | SUB )*>

$<$ !ATTLIST A HREF CDATA \#REQUIRED >

$<$ !-- SUP and SUB are superscripts and subscripts. In our content model, they are allowed to contain additional SUP and SUB elements, although we may decide to restrict them to PCDATA at some point -->

$<$ !ELEMENT SUP ( \#PCDATA | A | BR | SUP | SUB )* > $<$ !ELEMENT SUB ( \#PCDATA | A | BR | SUP | SUB )*>

\section{Appendix B}

A sample text file from the ADS Abstract Service showing XML markup for the full bibliographic entry, including records from STI, $M N R A S$, and SIMBAD. Items in bold are those selected to create the canonical text file shown in Appendix C.

$<$ ? xml version= "1.0"? $>$

$<$ !DOCTYPE ADS_BIBALL SYSTEM "ads.dtd"> $<$ ADS_BIBALL $>$

$<$ BIBRECORD origin= "STI" $>$

$<$ TITLE $>$ Spectroscopic confirmation of redshifts predicted by gravitational lensing $</$ TITLE $>$

$<$ AUTHORS $>$

$<\mathrm{AU}$ AF $=" 1 ">$

$<$ FNAME $>$ Tim $</$ FNAME $>$

$<$ LNAME $>$ Ebbels $</$ LNAME $>$

$</$ AU $>$

$<\mathrm{AU}$ AF="1" $>$

$<$ FNAME $>$ Richard $</$ FNAME $>$

$<$ LNAME $>$ Ellis $</$ LNAME $>$

$</ \mathrm{AU}>$

$<\mathrm{AU}$ AF $=$ "2" $>$

$<$ FNAME $>$ Jean-Paul $</$ FNAME $>$

$<$ LNAME $>$ Kneib $</$ LNAME $>$

$</ \mathrm{AU}>$

$<\mathrm{AU}$ AF $=$ "2" $>$

$<$ FNAME $>$ Jean-Francois $</$ FNAME $>$

$<$ LNAME $>$ LeBorgne $</$ LNAME $>$

$</ \mathrm{AU}>$

$<\mathrm{AU}$ AF $=$ "2" $>$

$<$ FNAME $>$ Roser $</$ FNAME $>$

$<$ LNAME $>$ Pello $</$ LNAME $>$

$</ \mathrm{AU}>$

$<\mathrm{AU}$ AF $=" 3 ">$

$<$ FNAME $>$ Ian $</$ FNAME $>$

$<$ LNAME $>$ Smail $</$ LNAME $>$
$</ \mathrm{AU}>$

$<\mathrm{AU}$ AF $=$ "4" $>$

$<$ FNAME $>$ Blai $</$ FNAME $>$

$<$ LNAME $>$ Sanahuja $</$ LNAME $>$

$</ \mathrm{AU}>$

$</$ AUTHORS $>$

$<$ AFFILIATIONS $>$

$<$ AF ident="AF_1" $>$ Cambridge, Univ. $</ \mathrm{AF}>$

$<\mathrm{AF}$ ident= "AF_2" $>$ Observatoire Midi-Pyrenees $</ \mathrm{AF}>$

$<$ AF ident="AF_3" $>$ Durham, Univ. $</ \mathrm{AF}>$

$<$ AF ident= "AF_4" $>$ Barcelona, Univ. $</$ AF $>$

$</$ AFFILIATIONS $>$

$<$ MSTRING $>$ Royal Astronomical Society, Monthly Notices, vol. 295 , p. $75</$ MSTRING $>$

$<$ MONOGRAPH $>$

$<$ MTITLE $>$ Royal Astronomical Society, Monthly

Notices $</$ MTITLE $>$

$<$ VOLUME $>295</$ VOLUME $>$

$</$ MONOGRAPH $>$

$<$ PAGE $>75</$ PAGE $>$

$<$ PUBDATE $>$

$<$ YEAR $>1998</$ YEAR $>$

$<$ MONTH $>03</$ MONTH $>$

$<$ /PUBDATE $>$

$<$ CATEGORIES $>$

$<$ CA $>$ Astrophysics $</$ CA $>$

$<$ CATEGORIES $>$

$<$ BIBCODE $>$ 1998MNRAS.295...75E $<$ /BIBCODE $>$

$<$ BIBTYPE $>$ article $</$ BIBTYPE $>$

$<$ IDENTIFIERS $>$

$<$ ID type $=$ "ACCNO" $>$ A98-51106 $</$ ID $>$

$<$ IDENTIFIERS $>$

$<$ KEYWORDS system="STI" $>$

$<$ KW $>$ GRAVITATIONAL LENSES $</$ KW $>$

$<$ KW $>$ RED SHIFT $</$ KW $>$

$<$ KW $>$ HUBBLE SPACE TELESCOPE $</$ KW $>$

$<$ KW $>$ GALACTIC CLUSTERS $</$ KW $>$

$<$ KW $>$ ASTRONOMICAL SPECTROSCOPY $</$ KW $>$

$<$ KW $>$ MASS DISTRIBUTION $</$ KW $>$

$<$ KW $>$ SPECTROGRAPHS $</$ KW $>$

$<$ KW $>$ PREDICTION ANALYSIS TECHNIQUES $</$ KW $>$

$<$ KW $>$ ASTRONOMICAL PHOTOMETRY $</$ KW $>$

$</$ KEYWORDS $>$

$<$ ABSTRACT $>$

We present deep spectroscopic measurements of 18 distant field galaxies identified as gravitationally lensed arcs in a Hubble Space Telescope image of the cluster Abell 2218. Redshifts of these objects were predicted by Kneib et al. using a lensing analysis constrained by the properties of two bright arcs of known redshift and other multiply imaged sources. The new spectroscopic identifications were obtained using long exposures with the LDSS-2 spectrograph on the William Herschel Telescope, and demonstrate the capability of that instrument to reach new limits, $R=24$; the lensing magnification implies true source magnitudes as faint as $R=25$. Statistically, our 
measured redshifts are in excellent agreement with those predicted from Kneib et al.'s lensing analysis, and this gives considerable support to the redshift distribution derived by the lensing inversion method for the more numerous and fainter arclets extending to $R=25.5$. We explore the remaining uncertainties arising from both the mass distribution in the central regions of Abell 2218 and the inversion method itself, and conclude that the mean redshift of the faint field population at $R=25.5$ $(B=26-27)$ is low, $(z=0.8-1)$. We discuss this result in the context of redshift distributions estimated from multicolor photometry.

$<$ ABSTRACT $>$

$<$ BIBRECORD $>$

\section{$<$ BIBRECORD origin="MNRAS" $>$}

$<$ TITLE $>$ Spectroscopic confirmation of redshifts predicted by gravitational lensing $</$ TITLE $>$

$<$ AUTHORS $>$

$<\mathrm{AU}$ AF $=$ "1" $>$

$<$ FNAME $>$ Tim $</$ FNAME $>$

$<$ LNAME $>$ Ebbels $</$ LNAME $>$

$</ \mathbf{A U}>$

$<$ AU AF ="1" EM="1" $>$

$<$ FNAME $>$ Richard $</$ FNAME $>$

$<$ LNAME $>$ Ellis $</$ LNAME $>$

$</$ AU $>$

$<\mathrm{AU}$ AF $=" 2 ">$

$<$ FNAME $>$ Jean-Paul $<$ /FNAME $>$

$<$ LNAME $>$ Kneib $</$ LNAME $>$

$</ \mathbf{A U}>$

$<$ AU AF $=" 2 ">$

$<$ FNAME $>$ Jean-Fran\&ccedil;ois $</$ FNAME $>$

$<$ LNAME $>$ LeBorgne $</$ LNAME $>$

$</ \mathbf{A U}>$

$<\mathrm{AU}$ AF ="2" $>$

$<$ FNAME $>$ Roser $</$ FNAME $>$

$<$ LNAME $>$ Pell\&oacute; $</$ LNAME $>$

$</ \mathbf{A U}>$

$<$ AU AF $=$ "3" $>$

$<$ FNAME $>$ Ian $</$ FNAME $>$

$<$ LNAME $>$ Smail $</$ LNAME $>$

$</ \mathbf{A U}>$

$<$ AU AF ="4" $>$

$<$ FNAME $>$ Blai $</$ FNAME $>$

$<$ LNAME $>$ Sanahuja $</$ LNAME $>$

$</ \mathbf{A U}>$

$</$ AUTHORS $>$

$<$ AFFILIATIONS $>$

$<$ AF ident="AF_1" $>$ Institute of Astronomy, Madingley Road, Cambridge CB3 0HA $</$ AF $>$

$<$ AF ident="AF_2" $>$ Observatoire Midi-Pyr\&eacute;n\&eacute; es, 14 Avenue E. Belin $</ \mathbf{A F}>$

$<$ AF ident="AF_3" $>$ Department of Physics, University of Durham, South Road, Durham DH1 3LE $</$ AF $>$

$<$ AF ident="AF_4" $>$ Departament d\&apos;Astronomia i
Meteorologia, Universitat de Barcelona, Diagonal 648, 08028 Barcelona, Spain $</ \mathbf{A F}>$

$</$ AFFILIATIONS $>$

$<$ EMAILS $>$

$<$ EM ident="EM_1" $>$ rse@ ast.cam.ac.uk $</$ EM $>$

$</$ EMAILS $>$

$<$ MSTRING $>$ Monthly Notices of the Royal Astronomical

Society, Volume 295, Issue 1, pp. 75-91. $<$ MSTRING $>$

$<$ MONOGRAPH $>$

$<$ MTITLE $>$ Monthly Notices of the Royal Astronomical

Society $</$ MTITLE $>$

$<$ MTITLE $>$ Monthly Notices of the Royal Astronomical

Society $</$ MTITLE $>$

$<$ VOLUME $>\mathbf{2 9 5}</$ VOLUME $>$

$<$ ISSUE $>1</$ ISSUE $>$

$<$ MONOGRAPH $>$

$<$ PAGE $>$ 75 $</$ PAGE $>$

$<$ LPAGE $>$ 91 $</$ LPAGE $>$

$<$ PUBDATE $>$

$<$ YEAR $>1998</$ YEAR $>$

$<$ MONTH $>03</$ MONTH $>$

$<$ PUBDATE $>$

$<$ COPYRIGHT $>$ 1998: The Royal Astronomical

Society $</$ COPYRIGHT $>$

$<$ BIBCODE $>1998$ MNRAS.295 ...75E < /BIBCODE $>$

$<$ KEYWORDS system="AAS" $>$

$<$ KW > GALAXIES: CLUSTERS: INDIVIDUAL: ABELL $2218</$ KW $>$

$<$ KW $>$ GALAXIES: EVOLUTION $</$ KW $>$

$<$ KW $>$ COSMOLOGY: OBSERVATIONS $</$ KW $>$

$<$ KW $>$ GRAVITATIONAL LENSING $</$ KW $>$

$</$ KEYWORDS $>$

$<$ ABSTRACT $>$

We present deep spectroscopic measurements of 18 distant field galaxies identified as gravitationally lensed arcs in a Hubble Space Telescope image of the cluster Abell2218. Redshifts of these objects were predicted by Kneib et al. using a lensing analysis constrained by the properties of two bright arcs of known redshift and other multiply imaged sources. The new spectroscopic identifications were obtained using long exposures with the LDSS-2 spectrograph on the William Herschel Telescope, and demonstrate the capability of that instrument to reach new limits, R\&sime;24 the lensing magnification implies true source magnitudes as faint as R\&sime;25. Statistically, our measured redshifts are in excellent agreement with those predicted from Kneib et al.'s lensing analysis, and this gives considerable support to the redshift distribution derived by the lensing inversion method for the more numerous and fainter arclets extending to $R \&$ sime;25.5. We explore the remaining uncertainties arising from both the mass distribution in the central regions of Abell2218 and the inversion method itself, and conclude that the mean redshift of the faint field population at R\&sime;25.5 (B\&sim;26\&ndash;27) is low, \&lang;z\&rang;=0.8\&ndash;1. We discuss this result 
in the context of redshift distributions estimated from multicolour photometry. Although such comparisons are not straightforward, we suggest that photometric techniques may achieve a reasonable level of agreement, particularly when they include near-infrared photometry with discriminatory capabilities in the $1 \& l t ; z \& l t ; 2$ range. $</$ ABSTRACT $>$

$</$ BIBRECORD $>$

$<$ BIBRECORD origin="SIMBAD" $>$

$<$ TITLE $>$ Spectroscopic confirmation of redshifts predicted by gravitational lensing. $</$ TITLE $>$

$<$ AUTHORS $>$

$<\mathrm{AU}>$

$<$ FNAME $>$ T. $</$ FNAME $>$

$<$ LNAME $>$ Ebbels $</$ LNAME $>$

$</ \mathrm{AU}>$

$<\mathrm{AU}>$

$<$ FNAME $>$ R. $</$ FNAME $>$

$<$ LNAME $>$ Ellis $</$ LNAME $>$

$</ \mathrm{AU}>$

$<\mathrm{AU}>$

$<$ FNAME $>$ J.-P. $</$ FNAME $>$

$<$ LNAME $>$ Kneib $</$ LNAME $>$

$</$ AU $>$

$<\mathrm{AU}>$

$<$ FNAME $>$ J.-F. $</$ FNAME $>$

$<$ LNAME $>$ LeBorgne $</$ LNAME $>$

$</ \mathrm{AU}>$

$<\mathrm{AU}>$

$<$ FNAME $>$ R. $</$ FNAME $>$

$<$ LNAME $>$ Pell\&oacute $;</$ LNAME $>$

$</ \mathrm{AU}>$

$<\mathrm{AU}>$

$<$ FNAME $>$ I. $</$ FNAME $>$

$<$ LNAME $>$ Smail $</$ LNAME $>$

$</ \mathrm{AU}>$

$<\mathrm{AU}>$

$<$ FNAME $>$ B. $</$ FNAME $>$

$<$ LNAME $>$ Sanahuja $</$ LNAME $>$

$</ \mathrm{AU}>$

$</$ AUTHORS $>$

$<$ MSTRING $>$ Mon. Not. R. Astron. Soc., 295, 75-91 $(1998)</$ MSTRING $>$

$<$ MONOGRAPH $>$

$<$ MTITLE $>$ Mon. Not. R. Astron. Soc. $</$ MTITLE $>$

$<$ VOLUME $>295<$ /VOLUME $>$

$<$ MONOGRAPH $>$

$<$ PAGE $>75</$ PAGE $>$

$<$ LPAGE $>91</$ LPAGE $>$

$<$ PUBDATE $>$

$<$ YEAR $>1998</$ YEAR $>$

$<$ MONTH $>03</$ MONTH $>$

$</$ PUBDATE $>$

$<$ BIBCODE $>1998$ MNRAS.295...75E $<$ /BIBCODE $>$

$</$ BIBRECORD $>$
$</$ ADS_BIBALL $>$

\section{Appendix C}

An example of an extracted text file from the ADS Abstract Service showing only the preferred instances of each field in XML markup for same bibliographic entry listed in Appendix B.

$<$ ?xml version= "1.0"? $>$

$<$ !DOCTYPE ADS_ABSTRACT SYSTEM "ads.dtd" > $<$ ADS_ABSTRACT $>$

$<$ TITLE $>$ Spectroscopic confirmation of redshifts predicted by gravitational lensing $</$ TITLE $>$

$<$ AUTHORS $>$

$<\mathrm{AU}$ AF $=" 1 ">$

$<$ FNAME $>$ Tim $</$ FNAME $>$

$<$ LNAME $>$ Ebbels $</$ LNAME $>$

$</ \mathrm{AU}>$

$<\mathrm{AU}$ AF="1" $\mathrm{EM}=$ "1" $>$

$<$ FNAME $>$ Richard $</$ FNAME $>$

$<$ LNAME $>$ Ellis $</$ LNAME $>$

$</ \mathrm{AU}>$

$<\mathrm{AU}$ AF $=" 2 ">$

$<$ FNAME $>$ Jean-Paul $<$ /FNAME $>$

$<$ LNAME $>$ Kneib $</$ LNAME $>$

$</ \mathrm{AU}>$

$<\mathrm{AU}$ AF $=$ "2" $>$

$<$ FNAME $>$ Jean-Fran\&ccedil;ois $</$ FNAME $>$

$<$ LNAME $>$ LeBorgne $</$ LNAME $>$

$</ \mathrm{AU}>$

$<\mathrm{AU}$ AF $=$ "2" $>$

$<$ FNAME $>$ Roser $</$ FNAME $>$

$<$ LNAME $>$ Pell\&oacute $;</$ LNAME $>$

$</ \mathrm{AU}>$

$<\mathrm{AU} \mathrm{AF}=$ "3" $>$

$<$ FNAME $>$ Ian $</$ FNAME $>$

$<$ LNAME $>$ Smail $</$ LNAME $>$

$</ \mathrm{AU}>$

$<\mathrm{AU}$ AF $=$ "4" $>$

$<$ FNAME $>$ Blai $</$ FNAME $>$

$<$ LNAME $>$ Sanahuja $</$ LNAME $>$

$</ \mathrm{AU}>$

$</$ AUTHORS $>$

$<$ AFFILIATIONS $>$

$<\mathrm{AF}$ ident= "AF_1" $>$ Institute of Astronomy, Madingley

Road, Cambridge CB3 0HA $</ \mathrm{AF}>$

$<$ AF ident= "AF_2" $>$ Observatoire Midi-Pyr\&eacute;

n\&eacute;es, 14 Avenue E. Belin $</ \mathrm{AF}>$

$<\mathrm{AF}$ ident="AF_3" $>$ Department of Physics, University of Durham, South Road, Durham DH1 3LE $</ \mathrm{AF}>$

$<\mathrm{AF}$ ident="AF_4" $>$ Departament d\&apos;Astronomia i Meteorologia, Universitat de Barcelona, Diagonal 648, 08028 Barcelona, Spain $</ \mathrm{AF}>$ 


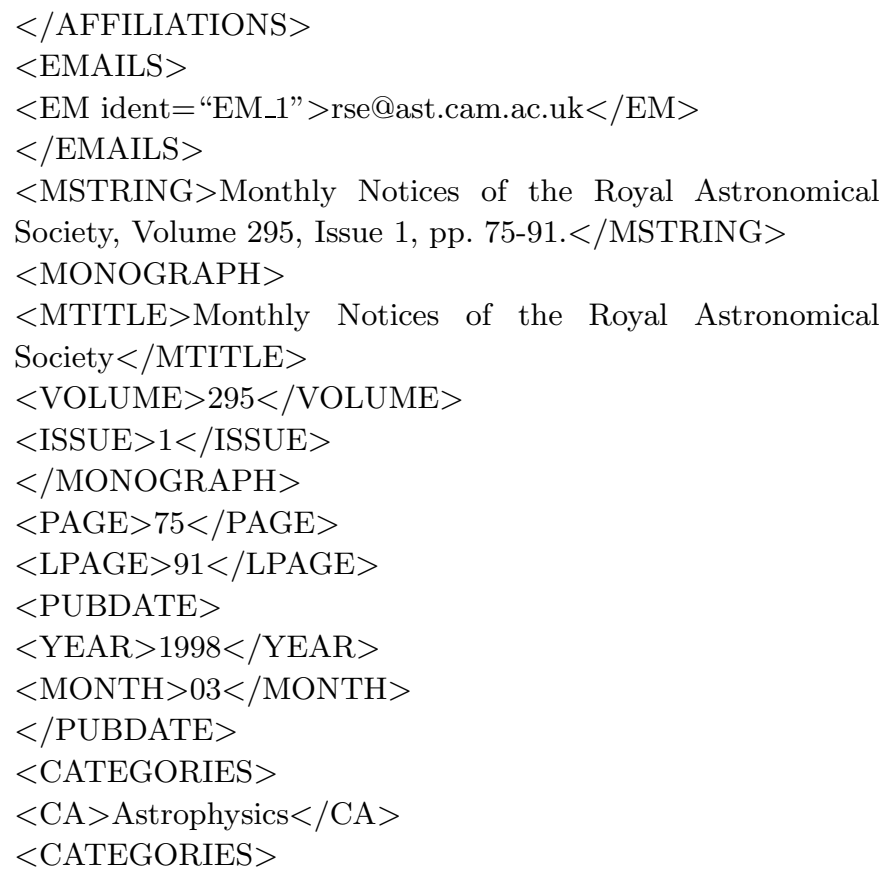
arcs in a Hubble Space Telescope image of the cluster Abell2218. Redshifts of these objects were predicted by
Kneib et al. using a lensing analysis constrained by the properties of two bright arcs of known redshift and other multiply imaged sources. The new spectroscopic identifications were obtained using long exposures with the LDSS-2 spectrograph on the William Herschel Telescope, and demonstrate the capability of that instrument to reach new limits, R\&sime;24 the lensing magnification implies true source magnitudes as faint as R\&sime;25. Statistically, our measured redshifts are in excellent agreement with those predicted from Kneib et al.'s lensing analysis, and this gives considerable support to the redshift distribution derived by the lensing inversion method for the more numerous and fainter arclets extending to R\&sime;25.5. We explore the remaining uncertainties arising from both the mass distribution in the central regions of Abell2218 and the inversion method itself, and conclude that the mean redshift of the faint field population at R\&sime;25.5 (B\&sim;26\&ndash;27) is low, \&lang;z\&rang;=0.8\&ndash;1. We discuss this result in the context of redshift distributions estimated from multicolour photometry. Although such comparisons are not straightforward, we suggest that photometric techniques may achieve a reasonable level of agreement, particularly when they include near-infrared photometry with discriminatory capabilities in the 1\&lt;z\&lt;2 range. $</$ ABSTRACT $>$

$</$ ADS_ABSTRACT $>$

\section{References}

Abt H.A., 1992, PASP 104, 235

Abt H.A., 1994, PASP 106, 1015

Abt H.A., 1995, ApJ 455, 407

Accomazzi A., Eichhorn G., Grant C.S., Kurtz M.J., Murray S.S., 2000, A\&AS 143, 85

Accomazzi A., Eichhorn G., Kurtz M.J., Grant C.S., Murray S.S., 1998, ASP Conf. Ser. 172: Astronomical Data Analysis Software and Systems VIII, p. 291

American Astronomical Society Manuscript Preparation, 1999, http://www.journals.uchicago.edu/AAS/AASTeX

Boyce P.B., 1998, ASP Conf. Ser. 153: Library and Information Services in Astronomy III, p. 107

Boyce P.B., Biemesderfer C., 1996, ASP Conf. Ser. 101: Astronomical Data Analysis Software and Systems V, p. 547

Egret D., Wenger M., 1988, ESO Conf. \#28: Astronomy from Large Databases, Murtagh F. and Heck A. (eds.), p. 323

Eichhorn G., Kurtz M.J., Accomazzi A., Grant C.S., Murray S.S., 2000, A\&AS 143, 61

Goldfarb C.F., Rubinsky Y., 1991, The Sgml Handbook. Clarendon Press

Harold E.R., 1998, Xml: Extensible Markup Language. IDG Books Worldwide

Helou G., Madore B., 1988, ESO Conf. \#28: Astronomy from Large Databases, Murtagh F. and Heck A. (eds.), p. 335

International Organization for Standardization 1987, Information and Processing - 8-bit Single-byte Coded Graphic Character Sets, Geneva 
Jacobsen D., 1996, http://www.ecst.csuchico.edu/ ${ }^{\text {jacobsd }}$ /bib/formats/refer.html

Knuth D.E., 1984, The TeXbook. Addison-Wesley Publishing Co.

Kurtz M.J., Eichhorn G., Accomazzi A., Grant C.S., Murray S.S., Watson J.M., 2000, A\&AS 143, 41

Lamport L., 1986, LaTex: A Documentation Preparation System. Addison-Wesley Publishing Co.

Lee J., Dubin D.S., Kurtz M.J., 1999, ASP Conf. Ser. 172: Astronomical Data Analysis Software and Systems VIII, p. 287

Los Alamos National Laboratory 1991, http://xxx.lanl.gov

Powell T.A., Whitworth D., 1998, Html: Programmer's Reference. Osborne McGraw-Hill

Schmadel L.D., 1979, Bulletin d'Information du Centre de Données Stellaires 17, 2

Schmadel L.D., 1982, IAU Colloq. 64: Automated Data Retrieval in Astronomy, p. 159

Schmadel L.D., 1989, ASP Conf. Ser. 153: Library and Information Services in Astronomy III, p. 77

Schmitz M., Helou G., Dubois P., Lague C., Madore B., Corwin
H.G.J., Lesteven S., 1995, Vistas Astron. 39, 272

Schulman E., French J.C., Powell A.L., Eichhorn G., Kurtz M.J., Murray S.S., 1997, PASP 109, 1278

Unicode Consortium, 1996, The Unicode Standard: Version 2.0. Addison-Wesley Pub. Co.

van Steenberg M., 1992, Desktop Publishing in Astronomy \& Space Sciences, Heck A. (ed.). Singapore, World Scientific, p. 143

van Steenberg M., Gass J., Brotzman L.E., Warnock A., Kovalsky D., Giovane F., 1992, Newslett. Am. Astron. Soc. 62,11

Wall L., Schwartz R.L., 1991, Programming PERL. O'Reilly \& Associates, Inc.

Warnock A., Gass J., Brotzman L.E., van Steenberg M.E., Kovalsky D., Giovane F., 1992, Newslett. Am. Astron. Soc. 62,10

Warnock A., van Steenberg M.E., Brotzman L.E., Gass J.E., Kovalsky D., Giovane F., 1993, ASP Conf. Ser. 52: Astronomical Data Analysis Software and Systems II, p. 137 